\title{
GLSM realizations of maps and intersections of Grassmannians and Pfaffians
}

\author{
Andrei Căldăraru, ${ }^{a}$ Johanna Knapp ${ }^{b}$ and Eric Sharpe ${ }^{c}$ \\ ${ }^{a}$ Department of Mathematics, University of Wisconsin, \\ 480 Lincoln Dr., Madison, WI 53706, U.S.A. \\ ${ }^{b}$ Mathematical Physics Group, University of Vienna, \\ Boltzmanngasse 5, 1090 Vienna, Austria \\ ${ }^{c}$ Department of Physics, Virginia Tech, \\ 850 West Campus Dr.,Blacksburg, VA 24061, U.S.A. \\ E-mail: andreic@math.wisc.edu, johanna.knapp@univie.ac.at, \\ ersharpe@vt.edu
}

ABSTRACT: In this paper we give gauged linear sigma model (GLSM) realizations of a number of geometries not previously presented in GLSMs. We begin by describing GLSM realizations of maps including Veronese and Segre embeddings, which can be applied to give GLSMs explicitly describing non-complete intersection constructions such as the intersection of one hypersurface with the image under some map of another. We also discuss GLSMs for intersections of Grassmannians and Pfaffians with one another, and with their images under various maps, which sometimes form exotic constructions of Calabi-Yaus, as well as GLSMs for other exotic Calabi-Yau constructions of Kanazawa. Much of this paper focuses on a specific set of examples of GLSMs for intersections of Grassmannians $G(2, N)$ with themselves after a linear rotation, including the Calabi-Yau case $N=5$. One phase of the GLSM realizes an intersection of two Grassmannians, the other phase realizes an intersection of two Pfaffians. The GLSM has two nonabelian factors in its gauge group, and we consider dualities in those factors. In both the original GLSM and a double-dual, one geometric phase is realized perturbatively (as the critical locus of a superpotential), and the other via quantum effects. Dualizing on a single gauge group factor yields a model in which each geometry is realized through a simultaneous combination of perturbative and quantum effects.

Keywords: Differential and Algebraic Geometry, Field Theories in Lower Dimensions, Supersymmetric Gauge Theory

ARXIV EPRINT: 1711.00047 


\section{Contents}

1 Introduction $\quad 2$

2 GLSM realizations of various maps 3

2.1 Veronese embeddings 3

2.2 Segre embedding 9

2.3 Diagonal map 11

2.4 Nonabelian Veronese embedding $\quad 13$

$\begin{array}{lll}2.5 & \text { More general maps } & 14\end{array}$

$\begin{array}{ll}2.6 & \text { Another perspective on hypersurfaces } \\ \end{array}$

3 General aspects of intersections of Grassmannians and Pfaffians $\quad 15$

$\begin{array}{ll}3.1 \text { Warm-up: complete intersections of hypersurfaces } & 15\end{array}$

3.2 Intersections of Grassmannians 16

$\begin{array}{lll}3.2 .1 & \text { Self-intersection with linear rotation } & 16\end{array}$

3.2.2 Intersection with Veronese embedding 21

3.2.3 Intersection with Segre embedding 24

$\begin{array}{lll}3.3 & \text { Intersections of Pfaffians } & 25\end{array}$

3.3.1 PAX models $\quad 25$

3.3.2 PAXY models $\quad 27$

3.3.3 PAXY for antisymmetric matrices 28

4 Phases of GLSMs for self-intersection of $G(2, N)$ for $N$ odd 30

4.1 First GLSM 31

4.2 Dual description 33

$\begin{array}{lll}4.3 & \text { Dualization on a single factor } & 35\end{array}$

5 Other Kanazawa Calabi-Yaus $\quad 37$

$\begin{array}{lll}5.1 & Y_{5}, Y_{10} & 37\end{array}$

$\begin{array}{lll}5.1 .1 & Y_{10} & 39\end{array}$

$\begin{array}{lll}5.1 .2 & Y_{5} & 40\end{array}$

$\begin{array}{lll}5.2 & X_{9} & 41\end{array}$

6 Conclusions $\quad 42$ 


\section{Introduction}

Over the last decade, a number of works have appeared showing how to construct GLSMs for more exotic spaces than complete intersections in toric varieties, in both abelian and nonabelian theories. For example, there now exist constructions for Pfaffian varieties, both as perturbative critical loci as well as with nonperturbative methods, nonperturbative constructions of branched double covers and noncommutative resolutions thereof, and GLSMs in which the geometric phases are not birational. Even though one expects a whole zoo of new examples, only a handful of explicit constructions have been given so far [6-14].

It is obvious that a greater variety of examples should exist. For instance, besides the Plücker embedding that plays a central role in the Grassmannian examples, there are further embeddings such as the Veronese embedding and the Segre embedding, whose images are not complete intersections (and hence not previously describable in GLSMs, save those which can be understood as Pfaffians). Part of the motivation of this work was to give a realization of these embeddings and non-complete intersections in terms of the GLSM. Furthermore, these embeddings can be used to realize a wide class of mathematical constructions of geometries which were not previously realizable in GLSMs, and we provide some simple illustrative examples.

A further motivation comes from recent constructions of exotic Calabi-Yau geometries in the mathematics literature that naturally generalize the known Grassmannian and Pfaffian constructions. One of these is an intersection of two Grassmannians in $\mathbb{P}^{9}$ and its homological projective dual [15] that has recently been shown to be a counter example to the birational Torelli problem $[16,17]$. In this work we give a GLSM realization of this pair of non-birational one-parameter Calabi-Yaus. We furthermore give a GLSM description of two Calabi-Yaus that are intersections of Pfaffian varieties with hypersurfaces [15].

In this work we consider GLSMs which reduce at low energies to non-linear sigma models on a certain geometry. In the Calabi-Yau case the FI couplings are not renormalized and there can be regions (phases) in the parameter space where the low-energy configuration of the GLSM is a non-linear sigma model with a Calabi-Yau target. In the Calabi-Yau case the FI parameters can be identified with Käher moduli of the Calabi-Yau inherited from the ambient space. In the non-Calabi-Yau case the FI coupling is renormalized, and its value is governed by a dynamically generated scale $\Lambda$. In this case the RG flows of the GLSM and nonlinear sigma model will asymptotically approach one another.

The GLSM is particularly useful in the Calabi-Yau case. Not only can does it give a physics realization of correspondences between Calabi-Yaus associated to different phases, it also encodes further information about the Calabi-Yau. For instance, the singularities in the Kähler moduli space are captured by Coulomb, or more generally the mixed, branches of the GLSM. In this work we will make use of these GLSM techniques to analyze examples of exotic Calabi-Yaus. As for the non-Calabi-Yau case, our main concern will be to identify GLSMs that reduce to non-linear sigma models on certain Fanos.

We begin in section 2 by describing GLSMs for (graphs of) functions such as Veronese and Segre embeddings, whose images are not complete intersections in general. For example, the image of the Veronese embedding of degree two is a determinantal variety, and we 
will see that in this special case the GLSM for the Veronese duplicates the PAXY model [9] for that determinantal variety. In section 3 we discuss GLSMs for various intersections of Grassmannians and Pfaffians. In section 4 we study phases of the GLSM for an intersection of Grassmannians, giving a physical realization of the Calabi-Yau geometry discussed in $[16,17]$. In our construction this Calabi-Yau arises as the $r \gg 0$ phase of a GLSM with gauge group $G=\frac{\mathrm{U}(2) \times \mathrm{U}(2)}{\mathrm{U}(1)}$, where $r$ is the FI parameter of the GLSM. The $r \ll 0$ phase of this GLSM realizes an intersection of Pfaffians, in a nonperturbative fashion, and we apply dualities which yield GLSMs in which the phases are realized in a variety of other fashions. Finally in section 5 we examine GLSMs for other exotic mathematical constructions of Calabi-Yaus discussed in [15].

\section{GLSM realizations of various maps}

\subsection{Veronese embeddings}

Recall a Veronese map of degree $d$ is a map $\nu: \mathbb{P} V \rightarrow \mathbb{P}\left(\operatorname{Sym}^{d} V\right)$ that sends homogeneous coordinates $\left[x_{0}, \cdots, x_{n}\right]$ (on an $n+1$-dimensional vector space $V$ ) to all possible monomials of degree $d$. For example, the degree two map $\mathbb{P}^{1} \rightarrow \mathbb{P}^{2}$ sends

$$
\left[x_{0}, x_{1}\right] \mapsto\left[x_{0}^{2}, x_{1}^{2}, x_{0} x_{1}\right] .
$$

If $\mathbb{P}(V)$ is a projective space of dimension $n$, then $\mathbb{P}\left(\operatorname{Sym}^{d} V\right)$ is a projective space of dimension

$$
\left(\begin{array}{c}
n+d \\
d
\end{array}\right)-1
$$

In this section we will describe how such maps are realized in GLSMs. In particular, the image of the Veronese embedding is not a complete intersection, and so its GLSM realization is of particular interest, as it provides access to GLSM realizations for a new class of mathematical constructions.

We can realize Veronese maps in GLSMs by adding a series of fields and superpotential terms to identify homogeneous coordinates in the image projective space with monomials of homogeneous coordinates in the original projective space. By integrating out the 'target' homogeneous coordinates, we are left with the original projective space. The images are non-complete-intersections. For degree two maps, the images are determinantal varieties, and in fact we shall see later that for degree two maps this construction is equivalent to the PAXY model of [9] for such determinantal varieties. In this fashion, we realize the Veronese embedding in a GLSM, or perhaps more accurately, its graph.

For example, consider the degree two map $\mathbb{P}^{1} \rightarrow \mathbb{P}^{2}$ above. Corresponding to this map, consider a GLSM defined by a U(1) gauge theory with chiral superfields ${ }^{1} x_{0,1}$, of charge 1 , $y_{0,1,2}$ of charge 2 , and $p_{0,1,2}$ of charge -2 , with superpotential

$$
W=p_{0}\left(y_{0}-x_{0}^{2}\right)+p_{1}\left(y_{1}-x_{1}^{2}\right)+p_{2}\left(y_{2}-x_{0} x_{1}\right) .
$$

\footnotetext{
${ }^{1}$ By a slight abuse of notation we use the same letters to denote superfields and their scalar components.
} 
The D-terms require that the collection $\left\{x_{0}, \cdots, y_{2}\right\}$ not be all zero in the phase in which $r \gg 0$, where $r$ is the FI parameter. The F-terms require

$$
y_{i}=\nu_{i}(x), \quad p_{i}=0, \quad \sum_{i} p_{i} \partial_{j} \nu_{i}(x)=0,
$$

where $\nu_{i}(x)$ denotes the image of the Veronese map. The $\mathrm{F}$ terms force $y_{i}$ to be the image of the Veronese embedding. Furthermore, the $\mathrm{F}$ terms for the $y_{i}$ 's immediately require all $p_{i}=0$, as expected to realize the desired geometry. (Mathematically, the Veronese embedding is always smooth, see e.g. [18, chapter 1.4], which is also consistent with the observation that the $p_{i}$ 's never become nonzero on vacua.)

Integrating out $y$ 's and $p$ 's reduces this model to just $\mathbb{P}^{1}$, realized with $x$ 's. (We will see that this is a special case of a more general result, for GLSM's realizing functions, or more properly graphs of functions, in section 2.5.)

More properly, this GLSM is realizing a locus in $\mathbb{P}_{[1,1,2,2,2]}^{4}$, where the last three homogeneous coordinates are identified with quadratic monomials in the first two. However, this locus is the same as a $\mathbb{P}^{1}$, parametrized by the first two homogeneous coordinates. As a quick consistency check, note that those first two homogeneous coordinates cannot both vanish: if they did, the other homogeneous coordinates would immediately vanish, and that point does not exist in the weighted projective space. For the same reason, this locus does not intersect the $\mathbb{Z}_{2}$ singularities of the weighted projective space, which arise where the first two homogeneous coordinates vanish.

Also note that if we define

$$
\begin{aligned}
& p_{00}=p_{0}, \quad p_{11}=p_{1}, \quad p_{10}=p_{01}=p_{2}, \\
& y_{00}=y_{0}, \quad y_{11}=y_{1}, \quad y_{10}=y_{01}=y_{2},
\end{aligned}
$$

then we can rewrite the potential in the form

$$
W=\sum_{i<j} p_{i j}\left(y_{i j}-x_{i} x_{j}\right)
$$

which has the same form as a symmetric-matrix version of the PAXY model discussed in $[9$, section 3.5$]$.

Let us take a moment to compare in greater detail to the pertinent PAXY model, for symmetric matrices, discussed in [9, section 3.5]. There, to describe the locus on which a symmetric $n \times n$ matrix $A(\phi)_{i j}$ has rank $k$, the authors introduced chiral superfields $p_{i j}$, an $O(k)$ gauge symmetry, a set of $n$ chiral superfields $x_{i a}$ valued in the fundamental, and a superpotential

$$
W=p_{i j}\left(A(\phi)_{i j}-x_{i a} x_{j a}\right) .
$$

In the present case, to describe the image of the Veronese embedding, we want to describe the locus on which the symmetric matrix $A_{i j}=y_{i j}$ has rank one, as the matrix $\left(x_{i} x_{j}\right)$ has $^{2}$ rank one. Following [9], the PAXY model is an $O(1)=\mathbb{Z}_{2}$ gauge theory with

\footnotetext{
${ }^{2}$ In general, for $\mathbb{P}^{n-1}$, so that there are $n$ homogeneous coordinates $x_{i}$, it is straightforward to show that the matrix $\left(x_{i} x_{j}\right)$ has only one nonvanishing eigenvalue, given by $\sum_{i} x_{i}^{2}$. Alternatively, to see that it has rank one, note that when multiplied into the vector $\left(a_{1}, a_{2}, \cdots, a_{n}\right)^{T}$, all components of the result are proportional to $\sum_{i} a_{i} x_{i}$.
} 
fields $x_{i}$ and a superpotential of the form

$$
W=p_{i j}\left(A(\phi)_{i j}-x_{i} x_{j}\right)
$$

(In the construction of [9], the $x_{i}$ are merely introduced to define the locus on which $A(\phi)$ has rank one; in the present context, however, they are homogeneous coordinates on the source projective space of the Veronese embedding.) Here, for $A(\phi)_{i j}=y_{i j}$, we have an identical superpotential. Furthermore, because the $y_{i j}$ are charge two and the $x_{i}$ charge one under the gauged $\mathrm{U}(1)$, that means the $y_{i j}$ are neutral under $\mathbb{Z}_{2} \subset \mathrm{U}(1)$, whereas the $x_{i}$ receive a sign flip, also matching [9].

In hindsight, the fact that the GLSM for the degree two Veronese model can be described as a PAXY model should not be a surprise, as the image of degree two Veronese embeddings are Pfaffian varieties (see e.g. [19, lecture 2]), hence describable by PAX and PAXY models.

Analogous descriptions extend to the images of Veronese embeddings of higher degree. For a degree $d$ map, label the homogeneous coordinates on the target projective space by $y_{i_{1} \cdots i_{d}}$, symmetric in its indices, then labeling the dual fields by $p_{i_{1} \cdots i_{d}}$ similarly, we can write the superpotential as

$$
W=p_{i_{1} \cdots i_{d}}\left(y_{i_{1} \cdots i_{d}}-x_{i_{1}} x_{i_{2}} \cdots x_{i_{d}}\right) .
$$

Just as there was a gauged $\mathbb{Z}_{2}$ in the degree two case, matching [9], here there is analogously a gauged $\mathbb{Z}_{d}$, corresponding to a subgroup of the U(1) gauge symmetry under which the $y_{i_{1} \cdots i_{d}}$ are neutral but the $x_{i}$ are charged. This is an analogue of the PAXY model for a 'hyperdeterminantal' variety, as one would expect for the image of a Veronese map of higher degree.

In passing, note that the nonminimal charges of the $y$ 's, dictated by gauge invariance of the superpotential, also reflect the degree of the Veronese embedding. For a degree $d$ Veronese embedding, all the maps of the sigma model into the target projective space have degree divisible by $d$. Sigma models with restrictions on degrees of maps have been discussed in e.g. [20-23] and references therein, and in those references, indeed, GLSMs for such sigma models are described by nonminimal $U(1)$ charges, divisible by a factor corresponding to the divisibility properties of the maps, in exactly the form we see here.

Let us analyze the model for the degree $d$ Veronese embedding more systematically, following the same pattern as discussed for the PAXY model in [9, section 3.3]. First, D-terms describe the space of $y$ 's and $x$ 's as a weighted projective space $\mathbb{P}_{[1, \cdots, 1, d, \cdots, d]}$, of dimension

$$
n+\left(\begin{array}{c}
n+d \\
d
\end{array}\right) .
$$

The $p$ 's then form a vector bundle over this projective space, defined by the sum of

$$
\left(\begin{array}{c}
n+d \\
d
\end{array}\right)
$$


copies of $\mathcal{O}(-d)$. In this language, the GLSM is realizing a complete intersection in the weighted projective space $\mathbb{P}_{[1, \cdots, 1, d, \cdots, d]}$. For the same reasons discussed earlier, this complete intersection can never intersect the $\mathbb{Z}_{d}$ singularities in that weighted projective space. Any singularities would have to arise in the complete intersection itself, not from the ambient weighted projective space. However, there are no singularities in the complete intersection. Defining

$$
E_{i_{1} \cdots i_{d}}=y_{i_{1} \cdots i_{d}}-x_{i_{1}} \cdots x_{i_{d}}
$$

the derivatives

$$
\left.\frac{\partial E_{i_{1} \cdots i_{d}}}{\partial(x, y)}\right|_{X}
$$

form a set of sections of the normal bundle to the locus $X \equiv\left\{E_{i_{1} \cdots i_{d}}=0\right\}$, and their linear independence guarantees that the F-term conditions

$$
p_{i_{1} \cdots i_{d}} \frac{\partial E_{i_{1} \cdots i_{d}}}{\partial(x, y)}=0
$$

can only be solved by taking all the $p_{i_{1} \cdots i_{d}}=0$. Thus, the given GLSM represents the Veronese embedding of $\mathbb{P}^{n}$, as advertised.

As another consistency check, we verify below that the Calabi-Yau condition realized in such GLSMs by demanding that the one-loop correction to the Fayet-Iliopoulos parameter vanish, coincides with the mathematical condition to have a Calabi-Yau.

To see this, note that the sum of the $y_{i}$ charges above cancels the sum of the $p_{i}$ charges above, so that the sum of the charges in a GLSM realizing just the Veronese embedding is the same as the sum of the charges in the original projective space, the sum of the charges of the $x_{a}$ 's. Therefore, in order for the FI to be RG-invariant, one must take a hypersurface (or complete intersection) whose $\mathrm{U}(1)$ charge matches the sum of the charges of the $x_{a}$ 's. This is the same as the same as the Calabi-Yau condition.

To be concrete, consider the example of a degree three Veronese embedding $\mathbb{P}^{2} \rightarrow \mathbb{P}^{9}$ :

$$
\left[x_{0}, x_{1}, x_{2}\right] \mapsto\left[x_{0}^{3}, x_{1}^{3}, x_{2}^{3}, x_{1}^{2} x_{2}, x_{1}^{2} x_{3}, x_{2}^{2} x_{1}, x_{2}^{2} x_{3}, x_{3}^{2} x_{1}, x_{3}^{2} x_{2}, x_{1} x_{2} x_{3}\right] .
$$

To get a Calabi-Yau, we intersect the image with a hyperplane (a hypersurface of degree one) in $\mathbb{P}^{9}$. This may be counterintuitive, as when dealing with complete intersections, we work with the sum of the degrees, but this is not a complete intersection. To see more explicitly that the result is Calabi-Yau, let us consider an example of a degree one hypersurface in $\mathbb{P}^{9}$ intersecting the image of the embedding:

$$
y_{0}+y_{1}+y_{2}=x_{0}^{3}+x_{1}^{3}+x_{2}^{3} .
$$

Clearly, the intersection of a hyperplane in $\mathbb{P}^{9}$ with the image of the (degree 3 ) Veronese embedding is equivalent to a cubic in $\mathbb{P}^{2}$, which is Calabi-Yau.

To realize the example above in a GLSM, we consider a theory with gauge group U(1), and matter

- 3 chiral superfields $x_{i}$ of charge 1 , corresponding to homogeneous coordinates on the original $\mathbb{P}^{2}$, 
- 10 chiral superfields $y_{j}$ of charge 3 , corresponding to homogeneous coordinates on the image $\mathbb{P}^{9}$,

- 10 chiral superfields $p_{j}$, charge -3 , multiplying superpotential terms that identify the homogeneous coordinates of the $\mathbb{P}^{9}$ with the image of the Veronese embedding,

- one chiral superfield $q$ of charge -3 , realizing a hyperplane in $\mathbb{P}^{9}$,

and superpotential

$$
W=\sum_{j=0}^{9} p_{j}\left(y_{j}-\nu_{j}(x)\right)+q G(y),
$$

where $\nu_{j}(x)$ is the $j$ th entry in the Veronese embedding (e.g. $\nu_{0}(x)=x_{0}^{3}, \nu_{1}(x)=x_{1}^{3}$, and so forth), and $G(y)$ is linear in $y$ 's (hence gauge charge 3 ).

The F terms imply

$$
G(y)=0, \quad y_{j}=\nu_{j}(x), \quad \sum_{j} p_{j} \partial_{i} \nu_{j}(x)=0, \quad p_{j}+q \frac{\partial G}{\partial y_{j}}=0
$$

At any fixed (nonzero) vev of the $x$ 's (and hence $y$ 's), the last two equations form 13 linear homogeneous equations for 11 unknowns (the $p$ 's and $q$ ), hence generically one expects that $q=0$ and all the $p_{j}=0$, as expected for the geometric interpretation we have described.

Note that the sum of the U(1) charges is zero - the charges of the $p_{j}$ 's and $y_{j}$ 's cancel out, leaving one just with the charges of the $x_{i}$ 's and $q$. Thus, as expected, we see that our GLSM description of the Veronese embedding correctly realizes the Calabi-Yau condition, as the condition that the Fayet-Iliopoulos parameter does not run and so is RG-invariant.

So far we have discussed intersections of the image of the Veronese embedding with hypersurfaces in the target space. In principle, one can also discuss images of hypersurfaces under the Veronese embedding, as well as their intersections with hypersurfaces in the target. For example, let $f(x)$ define a hypersurface of degree $d_{f}$ in the (original) projective space defined by the $x$ 's, and $G(y)$ a hypersurface of degree $d_{G}$ in the target projective space, defined by the $y$ 's. Suppose the $x$ 's and $y$ 's are related by a Veronese embedding of degree $d$. We could describe the intersection of the image of $\{f(x)=0\}$ with $\{G(y)=0\}$ with a GLSM with gauge group U(1) and the following fields:

- chiral superfields $x_{i}$ of charge 1 , corresponding to homogeneous coordinates in the original projective space,

- chiral superfields $y_{j}$ of charge $d$, corresponding to homogeneous coordinates in the target projective space,

- chiral superfields $p_{j}$ of charge $-d$ enforcing the Veronese map,

- a chiral superfield $s$ of charge $-d_{f}$,

- a chiral superfield $q$ of charge $-d d_{G}$, 
and superpotential

$$
W=\sum_{j} p_{j}\left(y_{j}-\nu_{j}(x)\right)+s f(x)+q G(y) .
$$

The F-terms imply, for example,

$$
y_{j}=\nu_{j}(x), \quad f(x)=0, \quad G(y)=0,
$$

and in this fashion one sees that, in the $r \gg 0$ phase, this GLSM is describing the intersection of the image of $\{f(x)=0\}$ with $\{G(y)=0\}$.

For completeness, let us also consider the $r \ll 0$ limit of these models. To be concrete, consider a quadratic Veronese embedding, say $\mathbb{P}^{1} \rightarrow \mathbb{P}^{2}$. Here we have

- two chiral superfields $x_{i}$ of charge 1 ,

- three chiral superfields $y_{j}$ of charge 2 ,

- three chiral superfields $p_{j}$ of charge -2 ,

and superpotential

$$
W=p_{0}\left(y_{0}-x_{0}^{2}\right)+p_{1}\left(y_{1}-x_{1}^{2}\right)+p_{2}\left(y_{2}-x_{0} x_{1}\right) .
$$

For $r \ll 0$, D terms require that the $p$ 's are not all zero. At the same time, the $\mathrm{F}$ terms for the $y_{j}$ require that all the $p$ 's vanish. This is a contradiction - classically, there are no ${ }^{3}$ supersymmetric vacua for $r \ll 0$, just as in the ordinary $\mathbb{P}^{n}$ model. Taking into account quantum corrections, one finds that RG flow drives the theory to the Coulomb branch where it sees a collection of discrete Coulomb vacua, as is well-known.

More generally, we can now build a GLSM describing the intersection of the image of $k$ hypersurfaces in $\mathbb{P}^{n}$, under a degree $d$ Veronese embedding, with $m$ hypersurfaces in the target projective space, of dimension

$$
\left(\begin{array}{c}
n+d \\
d
\end{array}\right)-1
$$

The expected dimension of this space will be $n-k-m$. Suppose the $k$ hypersurfaces in $\mathbb{P}^{n}$ have degrees $D_{1}, \cdots, D_{k}$, and the $m$ hypersurfaces in the target projective space have

\footnotetext{
${ }^{3}$ In passing, note that if there were supersymmetric vacua, there might be an interesting structure. Write the superpotential as

$$
W=p_{j} y_{j}-x_{i} A^{i j}(p) x_{j},
$$

then $A^{i j}$ is a mass matrix for the $x$ 's, given by

$$
\left(A^{i j}\right)=\left[\begin{array}{cc}
p_{0} & 1 / 2 p_{2} \\
1 / 2 p_{2} & p_{1}
\end{array}\right] .
$$

Away from the locus $\{\operatorname{det} A=0\}$, all massless fields have charge \pm 2 , so we have a branched double cover of Tot $\mathcal{O}(-1)^{3} \rightarrow \mathbb{P}^{2}$, defined by the $p$ 's and $y$ 's, branched over this degree two locus in $\mathbb{P}^{2}$, with remaining superpotential terms $p_{j} y_{j}[7,20-22]$.
} 
degrees $E_{1}, \cdots, E_{m}$. The Calabi-Yau condition is

$$
\left(\sum_{i} D_{i}\right)+d\left(\sum_{j} E_{j}\right)=n+1 .
$$

The corresponding GLSM is a U(1) gauge theory with matter

- $n+1$ chiral superfields $x_{i}$ of charge 1 , corresponding to homogeneous coordinates on $\mathbb{P}^{n}$,

- $(n+d) \cdots(n+1) / d$ ! chiral superfields $y_{j}$ of charge $d$, corresponding to homogeneous coordinates on the target projective space,

- $(n+d) \cdots(n+1) / d$ ! chiral superfields $p_{j}$ of charge $-d$, multiplying superpotential terms describing the Veronese embedding,

- $k$ chiral superfields $q_{a}$ of charges $-D_{1}, \cdots,-D_{k}$,

- $m$ chiral superfields $r_{b}$ of charges $-d E_{1}, \cdots,-d E_{k}$,

with superpotential

$$
W=p_{j}\left(y_{j}-\nu_{j}(x)\right)+q_{a} f_{a}(x)+r_{b} g_{b}(y),
$$

where $f_{a}(x), g_{b}(y)$ are the homogeneous polynomials defining the hypersurfaces in $\mathbb{P}^{n}$ and in the target projective space. The Calabi-Yau condition follows immediately from the sum of the $\mathrm{U}(1)$ charges.

The reader should note that the spaces above are not new to the literature - these are simply the complete intersections $\mathbb{P}^{n}\left[D_{1}, \cdots, D_{k}, d E_{1}, \cdots, d E_{m}\right]$, described in an unusual fashion.

For one example, we can obtain a Calabi-Yau threefold as the intersection of the image of a hypersurface in $\mathbb{P}^{5}$ of degree 2 , under a Veronese embedding of degree 2 , with a hypersurface in the target projective space of degree 2 . This is equivalent to the complete intersection $\mathbb{P}^{5}[2,4]$.

There are several other closely analogous examples which are straightforward to realize in GLSMs, which we will describe in subsequent subsections. As the analysis is very similar to what we have just described, our descriptions will be brief.

\section{$2.2 \quad$ Segre embedding}

The Segre embedding can be realized in a similar fashion to Veronese embeddings. Recall the Segre map is the map

$$
\mathbb{P}^{k} \times \mathbb{P}^{n} \longrightarrow \mathbb{P}^{(k+1)(n+1)-1}
$$

as

$$
\left[x_{0}, \cdots, x_{k}\right] \times\left[y_{0}, \cdots, y_{n}\right] \mapsto\left[x_{0} y_{0}, x_{0} y_{1}, \cdots, x_{n} y_{n}\right],
$$

where the $x_{i}$ are homogeneous coordinates on $\mathbb{P}^{k}$ and the $y_{j}$ are homogeneous coordinates on $\mathbb{P}^{n}$. 
We can realize this map in a GLSM as follows. Consider a $\mathrm{U}(1) \times \mathrm{U}(1)$ gauge theory, with matter

- $k+1$ chiral superfields $x_{i}$ of charge $(1,0)$,

- $n+1$ chiral superfields $y_{j}$ of charge $(0,1)$,

- $(k+1)(n+1)$ chiral superfields $z_{a}$ of charge $(1,1)$,

- $(k+1)(n+1)$ chiral superfields $p_{a}$ of charge $(-1,-1)$, multiplying superpotential terms that force the $z_{a}$ 's to be identified with the image of the Segre embedding,

and superpotential

$$
W=\sum_{a} p_{a}\left(z_{a}-S_{a}(x, y)\right)
$$

where $S_{a}(x, y)$ gives the image of the Segre embedding, meaning $S_{0}=x_{0} y_{0}, S_{1}=x_{0} y_{1}$, and so forth.

The analysis of this GLSM follows much as before. In the phase defined by FayetIliopoulos parameters $\left(r_{1}, r_{2}\right)$ where $r_{1} \gg 0$ and $r_{2} \gg 0$, D-terms imply that not all the $x$ 's vanish and not all the $y$ 's vanish, as one would expect for a pair of projective spaces. F-terms require

$$
z_{a}=S_{a}(x, y), \quad p_{a}=0, \quad \sum_{a} p_{a} \frac{\partial S_{a}}{\partial x_{i}}=0=\sum_{a} p_{a} \frac{\partial S_{a}}{\partial y_{j}},
$$

so we immediately recover the desired geometry, as before.

For purposes of computing Calabi-Yau hypersurfaces in the image, note that the charges of the $z_{a}, p_{a}$ cancel out in the renormalization of the Fayet-Iliopoulos parameter, so that only the $x_{i}, y_{j}$ charges contribute to the determination of the Calabi-Yau condition, in exactly the same fashion as the Veronese embedding previously discussed.

As one simple example, the image of the Segre embedding $\mathbb{P}^{1} \times \mathbb{P}^{1} \rightarrow \mathbb{P}^{3}$ is a quadric hypersurface in $\mathbb{P}^{3}$, specifically the hypersurface $z_{0} z_{1}=z_{2} z_{3}$, which can be seen by identifying

$$
z_{0}=x_{0} y_{0}, \quad z_{1}=x_{1} y_{1}, \quad z_{2}=x_{1} y_{0}, \quad z_{3}=x_{0} y_{1} .
$$

We can slightly rewrite the GLSM above to emphasize another part of the mathematics. The image of a Segre embedding is a determinantal variety, which admits PAX and PAXY descriptions, and the description above is of the form of a PAXY model. Specifically, write the target-space homogeneous coordinates as $z_{i j}$, where the Segre embedding relates $z_{i j}=x_{i} y_{j}$. Then, the GLSM has a superpotential of the form

$$
W=p_{i j}\left(z_{i j}-x_{i} y_{j}\right) .
$$

Now, the image of the Segre embedding is the locus in $\mathbb{P}^{(k+1)(n+1)-1}$ given by the zero locus of the $2 \times 2$ minors of the matrix with components $z_{i j}$.

Now, as will be reviewed more extensively in section 3.3.2, the PAXY model [9] that describes the locus in $\mathbb{P}^{(k+1)(n+1)-1}$ where the $N \times M$ matrix $A(\phi)_{i j}$ has rank $m$ (corresponding to the vanishing of all $(m+1) \times(m+1)$ minors $)$ is defined by a $\mathrm{U}(m)$ gauge theory with 
- $N$ chiral superfields $x_{i a}$ in the fundamental of $\mathrm{U}(m)$,

- $M$ chiral superfields $y_{i}^{a}$ in the antifundamental of $\mathrm{U}(m)$,

- $N M$ chiral superfields $p_{i j}$ which are neutral under $\mathrm{U}(m)$,

with superpotential

$$
W=p_{i j}\left(A(\phi)_{i j}-x_{i a} y_{j}^{a}\right) .
$$

Here, to describe the image of the Segre embedding, we wish to restrict to the rank-one locus of the $(k+1) \times(n+1)$ matrix $z_{i j}$, and it is straightforward to check that the matter content and superpotential of the Segre realization match the PAXY model for this case, with the PAXY U(1) corresponding to the antidiagonal U(1) of the Segre realization's $\mathrm{U}(1) \times \mathrm{U}(1)$.

Thus, as a consistency check, we see that the description of the Segre embedding above duplicates the description of the image as a determinantal variety via a PAXY description.

\subsection{Diagonal map}

The map

$$
\Delta: \mathbb{P}^{n} \longrightarrow \mathbb{P}^{n} \times \mathbb{P}^{n}
$$

embeds $\mathbb{P}^{n}$ diagonally into the product of projective spaces.

The corresponding GLSM is a $\mathrm{U}(1)$ gauge theory with matter

- $n+1$ chiral superfields $x_{i}$ of charge 1 , corresponding to homogeneous coordinates on the source $\mathbb{P}^{n}$,

- $n+1$ chiral superfields $y_{i}$ of charge 1 , corresponding to homogeneous coordinates on one of the target factors,

- $n+1$ chiral superfields $z_{i}$ of charge 1 , corresponding to homogeneous coordinates on the other factor,

- $2(n+1)$ chiral superfields $p_{i}, q_{i}$ of charge -1 ,

and superpotential

$$
W=p_{i}\left(y_{i}-x_{i}\right)+q_{i}\left(z_{i}-x_{i}\right) .
$$

Briefly, on the one hand, if we integrate out the $y \mathrm{~s}$ and $z \mathrm{~s}$, we recover the original projective space, whereas if we integrate out $x \mathrm{~s}$, we find the superpotential

$$
W=p_{i}^{\prime}\left(y_{i}-z_{i}\right) .
$$

In more detail, for $r \gg 0$, D-terms imply that not all the $x \mathrm{~s}, y \mathrm{~s}$, and $z \mathrm{~s}$ vanish. F-terms then imply

$$
x_{i}=y_{i}=z_{i}, \quad p_{i}=q_{i}=0 .
$$


Mathematically, the composition of the Segre and diagonal maps is equivalent to the Veronese embedding of degree two. To see this, write $\mathbb{P}^{n}=\mathbb{P} V$ for a vector space $V$ of dimension $n+1$. Then

$$
\begin{aligned}
\Delta: \mathbb{P} V & \longrightarrow \mathbb{P} V \times \mathbb{P} V, \\
z & \mapsto(z, z),
\end{aligned}
$$

and

$$
\begin{aligned}
& \text { Segre }: \mathbb{P} V \times \mathbb{P} V \longrightarrow \mathbb{P}(V \otimes V), \\
&(x, y) \quad \mapsto x \otimes y .
\end{aligned}
$$

Thus, the composition is the map $\mathbb{P} V \rightarrow \mathbb{P}(V \otimes V)$ given by $z \mapsto z \otimes z$. However, this factors through $\mathbb{P}\left(\mathrm{Sym}^{2} V\right)$, so in particular

$$
\text { Segre } \circ \Delta=j \circ \nu_{2},
$$

where $j: \mathbb{P}\left(\operatorname{Sym}^{2} V\right) \rightarrow \mathbb{P}(V \otimes V)$.

Physically, we can represent the composition

$$
\text { Segre } \circ \Delta: \mathbb{P}^{n} \stackrel{\Delta}{\longrightarrow} \mathbb{P}^{n} \times \mathbb{P}^{n} \stackrel{\text { Segre }}{\longrightarrow} \mathbb{P}^{(n+1)^{2}-1}
$$

as follows. Consider a GLSM with gauge group U(1) and matter

- $n+1$ chiral superfields $x_{i}$ of charge 1 , corresponding to homogeneous coordinates on the first source $\mathbb{P}^{n}$,

- $n+1$ chiral superfields $y_{i}$ of charge 1 , corresponding to homogeneous coordinates on the one of the middle $\mathbb{P}^{n}$ factors,

- $n+1$ chiral superfields $z_{i}$ of charge 1 , corresponding to homogeneous coordinates on another of the middle $\mathbb{P}^{n}$ factors,

- $(n+1)^{2}$ chiral superfields $w_{i j}$ of charge 2 , corresponding to homogeneous coordinates on the final $\mathbb{P}^{n^{2}+2 n}$,

- $2(n+1)$ chiral superfields $p_{i}, q_{i}$ of charge -1 ,

- $(n+1)^{2}$ chiral superfields $s_{i j}$ of charge -2 ,

and superpotential

$$
W=p_{i}\left(y_{i}-x_{i}\right)+q_{i}\left(z_{i}-x_{i}\right)+s_{i j}\left(w_{i j}-y_{i} z_{i}\right) .
$$

After integrating out the $p$ 's, $q$ 's, $y$ 's, and $z$ 's, this becomes

$$
W=s_{i j}\left(w_{i j}-x_{i} x_{j}\right),
$$

which is explicitly the GLSM representing $j \circ \nu_{2}: \mathbb{P}^{n} \rightarrow \mathbb{P}^{n^{2}+2 n}$. 


\subsection{Nonabelian Veronese embedding}

There is an analogue of the Veronese embedding for Grassmannians, see e.g. [19, section 6.10], which sends

$$
\nu_{d}: G(k, n) \longrightarrow G\left(\left(\begin{array}{c}
k+d \\
d
\end{array}\right),\left(\begin{array}{c}
n+d \\
d
\end{array}\right)\right)
$$

We describe a conjecture ${ }^{4}$ below for how this can be realized in a GLSM. Consider a GLSM with gauge group $\mathrm{U}(k)$, and matter as follows:

- $n$ chiral superfields $\Phi_{i}^{a}$ in the representation $\mathbf{k}$ of the gauge group,

- $\left(\begin{array}{c}n+d \\ d\end{array}\right)$ chiral superfields $\tilde{\Phi}_{i_{1} \cdots i_{d}}^{a_{1} \cdots a_{d}}, i_{1} \leq \cdots \leq i_{d}, a_{1} \leq \cdots \leq a_{d}$ (symmetric in both sets of indices), in the representation $\mathrm{Sym}^{\mathbf{d}} \mathbf{k}$ of the gauge group,

- $\left(\begin{array}{c}n+d \\ d\end{array}\right)$ chiral superfields $p_{a_{1} \cdots a_{d}}^{i_{1} \cdots i_{d}}, i_{1} \leq \cdots \leq i_{d}, a_{1} \leq \cdots \leq a_{d}$ (symmetric in both sets of indices) in the representation $\operatorname{Sym}^{\mathbf{d}} \overline{\mathbf{k}}$ of the gauge group,

with superpotential

$$
W=\sum_{i_{1} \leq \cdots \leq i_{d}} p_{a_{1} \cdots a_{d}}^{i_{1} \cdots i_{d}}\left(\tilde{\Phi}_{i_{1} \cdots i_{d}}^{a_{1} \cdots a_{d}}-\operatorname{Sym} \Phi_{i_{1}}^{a_{1}} \cdots \Phi_{i_{d}}^{a_{d}}\right)
$$

The analysis of this model is much the same as in previous sections. Note for the purpose of defining Calabi-Yau hypersurfaces by the RG running of the Fayet-Iliopoulos parameter that the contributions from the $p$ 's and $\tilde{\Phi}$ 's cancel out, so that this behaves just like an ordinary Grassmannian $G(k, n)$, and furthermore that integrating out $\tilde{\Phi}$ 's yields the ordinary Grassmannian model, as expected.

One point of confusion is that in the image Grassmannian, we only take the symplectic quotient by $\mathrm{U}(k)$ not

$$
U\left(\begin{array}{c}
k+d \\
d
\end{array}\right)
$$

We have not checked carefully, but we do observe that this appears to be analogous to the fact in earlier Veronese embeddings that one quotiented by a different gauge group (a cover of $\mathrm{U}(1)$ or of $\mathrm{U}(k))$, corresponding to the fact that maps into the target space all had certain divisibility properties, as in e.g. [20-23]. Here, the analogous divisibility properties would be rather more complicated.

\footnotetext{
${ }^{4}$ As we will not use this example elsewhere, it has not been checked as thoroughly as other cases discussed in this paper.
} 


\subsection{More general maps}

Given any homogeneous map $f: \mathbb{P}^{n} \rightarrow \mathbb{P}^{m}$, by which we mean a set of $m+1$ homogeneous polynomials, we can similarly define a corresponding GLSM.

Suppose the components of the map $f$ all have degree $k$. Then, the corresponding GLSM is a U(1) gauge theory with

- $n+1$ chiral multiplets $x_{i}$ of charge 1 ,

- $m+1$ chiral multiplets $y_{j}$ of charge $k$,

- $m+1$ chiral multiplets $p_{j}$ of charge $-k$,

and superpotential

$$
W=p_{j}\left(y_{j}-f_{j}(x)\right) .
$$

Strictly speaking, this GLSM describes the graph of the function $f(x)$, which for a map $f: X \rightarrow Y$ is defined to be

$$
\{(x, y) \in X \times Y \mid y=f(x)\} .
$$

The graph of $f$ always embeds into $X \times Y$, so the graph is always isomorphic to $X$, mathematically. Physically, the same result follows from the fact that $p$ 's and $y$ 's can be integrated out. The graph will be isomorphic to the image of $f$ if and only if $f$ is an embedding.

For a trivial example, consider a constant map $f$ on $\mathbb{P}^{n}$. After absorbing the constant into a trivial field redefinition, the superpotential can be written

$$
W=p_{j}\left(y_{j}-0\right) .
$$

The $\mathrm{F}$ terms require that $p$ and $y$ vanish, leaving one just with a copy of the supersymmetric $\mathbb{P}^{n}$ model, matching the mathematical prediction.

In the cases discussed so far (Veronese, Segre, diagonal embeddings), because they are embeddings, the image of the map is isomorphic to the source, and so the graph is isomorphic to the image.

For completeness, let us briefly outline composition. Given a map $f: \mathbb{P}^{n} \rightarrow \mathbb{P}^{m}$ of degree $k$, and a map $g: \mathbb{P}^{m} \rightarrow \mathbb{P}^{r}$ of degree $\ell$, we take the GLSM corresponding to the composition to be a $\mathrm{U}(1)$ gauge theory with matter

- $n+1$ chiral multiplets $x_{i}$ of charge 1 ,

- $m+1$ chiral multiplets $y_{j}$ of charge $k$,

- $r+1$ chiral multiplets $z_{a}$ of charge $k \ell$,

- $m+1$ chiral multiplets $p_{j}$ of charge $-k$,

- $r+1$ chiral multiplets $q_{a}$ of charge $-k \ell$, 
and superpotential

$$
W=p_{j}\left(y_{j}-f_{j}(x)\right)+q_{a}\left(z_{a}-g_{a}(y)\right) .
$$

Now, in the expression above, we can trivially integrate out $y$ 's and $p$ 's, to get a $\mathrm{U}(1)$ gauge theory of $x$ 's, $z$ 's, and $q$ 's, with superpotential

$$
W=q_{a}\left(z_{a}-g_{a}(f(x))\right),
$$

trivially corresponding to the map $g \circ f$. For this reason, we can identify the GLSM above with the composition of the GLSM's for $f$ and $g$.

\subsection{Another perspective on hypersurfaces}

As an entertaining aside, we can describe the GLSM for an ordinary hypersurface in a projective space in the language of maps.

Specifically, first interpret the defining equation of a hypersurface, a homogeneous polynomial $f$ of degree $d$, as a map $f: \mathbb{P}^{n} \rightarrow \mathbb{C}$. Then, take the intersection with the origin of $\mathbb{C}$, effectively realizing $f^{-1}(0)$.

We can describe this in a GLSM as follows. Consider a U(1) gauge theory with matter

- $n+1$ chiral superfields $x_{i}$ of charge 1 , corresponding to homogeneous coordinates on $\mathbb{P}^{n}$,

- one chiral superfield $y$ of charge $d$, corresponding to the image of $f$,

- two chiral superfields $p, q$ of charge $d$,

and superpotential

$$
W=q(y-f(x))+p(y-0) .
$$

Integrating out $y$ and $q$, one recovers the standard GLSM for a hypersurface.

\section{General aspects of intersections of Grassmannians and Pfaffians}

\subsection{Warm-up: complete intersections of hypersurfaces}

This section will be concerned with novel GLSM descriptions of complete intersections of Grassmannians and Pfaffians, so as a warm-up, we will describe a pertinent presentation of a complete intersection of hypersurfaces in a projective space, to illustrate a basic idea we will use later.

Consider hypersurfaces in $\mathbb{P}^{n}$ defined by two homogeneous polynomials $G_{1}(\phi), G_{2}(\phi)$ of degrees $d_{1}, d_{2}$, respectively. Ordinarily, we would describe this by a GLSM with $n+1$ chiral superfields $\phi_{i}$ of charge 1 and two additional chiral superfields $p_{1}, p_{2}$ of charges $-d_{1}$, $-d_{2}$, respectively, with superpotential

$$
W=p_{1} G_{1}(\phi)+p_{2} G_{2}(\phi) .
$$

However, there is an alternative presentation that one could use instead. Consider an alternative theory with $2(n+1)$ chiral superfields $\varphi_{i}, \tilde{\varphi}_{i}(i \in\{1, \cdots, n+1\})$ of charge 1 , 
two chiral superfields $p_{1}, p_{2}$ of charges $-d_{1},-d_{2}$, respectively, as before, plus $n+1$ chiral superfields $b_{i}$ of charge -1 , with superpotential

$$
W=p_{1} G_{1}(\varphi)+p_{2} G_{2}(\tilde{\varphi})+\sum_{i=1}^{n+1} b_{i}\left(\varphi_{i}-\tilde{\varphi}_{i}\right) .
$$

In this case, $p_{1}$ multiplies the superpotential term corresponding to the hypersurface $\left\{G_{1}=0\right\}$ in the space of $\varphi$ 's, $p_{2}$ multiplies the superpotential term corresponding to the hypersurface $\left\{G_{2}=0\right\}$ in the space of $\tilde{\varphi}$ 's, and the b's multiply superpotential terms identifying homogeneous coordinates on the two copies of $\mathbb{P}^{n}$.

Let us check this more thoroughly. For $r \gg 0$, D terms require that $\left\{\varphi_{1}, \cdots, \tilde{\varphi}_{n+1}\right\}$ are not all simultaneously zero. $\mathrm{F}$ terms require

$$
G_{1}=0, \quad G_{2}=0, \quad \varphi_{i}-\tilde{\varphi}_{i}=0, \quad p_{1} \partial_{i} G_{1}+b_{i}=0, \quad p_{2} \partial_{i} G_{2}-b_{i}=0 .
$$

which requires

$$
G_{1}=0, \quad G_{2}=0, \quad \varphi_{i}-\tilde{\varphi}_{i}=0, \quad p_{1} \partial_{i} G_{1}(\varphi)+p_{2} \partial_{i} G_{2}(\tilde{\varphi})=0,
$$

which after collapsing $\tilde{\varphi} \rightarrow \varphi$ reduce to the same conditions as in ordinary presentations of complete intersections. Nondegeneracy of the complete intersection requires $p_{1}=p_{2}=0$ and so $b_{i}=0$.

This second description is overkill for the simple case of a complete intersection of hypersurfaces in a projective space, but will be a prototype for intersections of Grassmannians and Pfaffian varieties.

\subsection{Intersections of Grassmannians}

Next, consider a Grassmannian $G(k, N)$. This is described [24] by a $\mathrm{U}(k)$ gauge theory with $N$ chiral multiplets $\phi_{a}^{i}$ in the fundamental representation. It has a natural embedding into a projective space of dimension

$$
\left(\begin{array}{c}
N \\
k
\end{array}\right)-1
$$

defined by the $\mathrm{SU}(k)$-invariant combinations of chiral superfields

$$
B^{i_{1} \cdots i_{k}}=\phi_{a_{1}}^{i_{1}} \cdots \phi_{a_{k}}^{i_{k}} \epsilon^{a_{1} \cdots a_{k}}
$$

These act as homogeneous coordinates on the projective space, and define what is known mathematically as the Plücker embedding.

\subsubsection{Self-intersection with linear rotation}

Given a Grassmannian $G(k, N)$, one can deform the Plucker embedding by replacing the baryons with linear combinations, and then intersect the deformed image with the original Plücker embedding. This is what we refer to as a 'self-intersection of Grassmannians,' the 
intersection of $G(k, N)$ with a linear deformation in its Plücker embedding. We will restrict to the case that $N$ odd in the following.

The expected dimension of this intersection is ${ }^{5}$

$$
2 k(N-k)+1-\left(\begin{array}{c}
N \\
k
\end{array}\right) .
$$

Furthermore, if the expected dimension is negative, then the intersection will be empty, so to have a nonempty intersection, one must require

$$
2 k(N-k)+1 \geq\left(\begin{array}{c}
N \\
k
\end{array}\right) .
$$

As a result, these intersections will typically be of interest only for relatively small values of $N$ and $k$.

The intersection will be smooth when the two Grassmannians intersect transversely, which will happen (for positive expected dimension) for generic rotations of one copy. Note however that for trivial rotations, equal to the identity, the dimension of the intersection jumps, so that the family is not flat at this point. (We will see this reflected in the occurrence of noncompact branches in the GLSM at such points.)

This intersection will be Calabi-Yau when

$$
\left(\begin{array}{l}
N \\
k
\end{array}\right)=2 N .
$$

We can see this as follows. Let $\mathbb{P}$ denote the projective space in which each copy of $G(k, N)$ is embedded, and $X$ the intersection of the two copies. For either Grassmannian $G(k, N)$, the adjunction formula says that

$$
K_{G}=\left.K_{\mathbb{P}}\right|_{G}+c_{1}\left(N_{G / \mathbb{P}}\right),
$$

where $G$ denotes the Grassmannian, and for any $X, K_{X}$ denotes $c_{1}$ of the canonical bundle of $X$. Now, $T G=S^{*} \otimes Q$, for $S$ the universal subbundle (rank $k$ ) and $Q$ the universal quotient bundle $(\operatorname{rank} N-k)$. Since $c_{1}(S)=\mathcal{O}(-1), c_{1}(Q)=\mathcal{O}(+1)$, we deduce $c_{1}(T G)=$ $(N-k) c_{1}\left(S^{*}\right)+k c_{1}(Q)=\mathcal{O}(N)$. We then have

$$
K_{G}=-N, \quad K_{\mathbb{P}}=-\left(\begin{array}{c}
N \\
k
\end{array}\right),
$$

\footnotetext{
${ }^{5}$ First note that the image of $G(k, N)$ in its Plücker embedding is locally described by

$$
\left(\begin{array}{c}
N \\
k
\end{array}\right)-1-k(N-k)
$$

equations. The expected dimension of the intersection is the dimension of the ambient space minus the number of equations, which for a general linear deformation, will be twice the number of equations above,
} hence the expected dimension is

$$
\left(\begin{array}{l}
N \\
k
\end{array}\right)-1-2\left[\left(\begin{array}{l}
N \\
k
\end{array}\right)-1-k(N-k)\right]=2 k(N-k)+1-\left(\begin{array}{l}
N \\
k
\end{array}\right)
$$


hence

$$
c_{1}\left(N_{G / \mathbb{P}}\right)=\left(\begin{array}{c}
N \\
k
\end{array}\right)-N
$$

If the intersection $X$ is transversal, then $\left.N_{X / G_{1}} \cong N_{G_{2} / \mathbb{P}}\right|_{X}$, where $G_{1}$ and $G_{2}$ denotes the two Grassmannians in $\mathbb{P}$. From the adjunction formula,

$$
K_{X}=\left.K_{G_{1}}\right|_{X}+c_{1}\left(N_{X / G_{1}}\right)=\left.K_{G_{1}}\right|_{X}+c_{1}\left(\left.N_{G_{2} / \mathbb{P}}\right|_{X}\right)=\left(\begin{array}{c}
N \\
k
\end{array}\right)-2 N .
$$

Hence, we recover condition (3.11) for the intersection to be Calabi-Yau. It is straightforward to check that only in the cases $N=5$ and $k=2$ or $k=3$ will this intersection be Calabi-Yau.

We can describe this intersection of $G(k, N)$ with itself in a GLSM as follows. Take a

$$
\frac{\mathrm{U}(1) \times \mathrm{SU}(k) \times \mathrm{SU}(k)}{\mathbb{Z}_{k} \times \mathbb{Z}_{k}}
$$

gauge theory, where each $\mathbb{Z}_{k}$ acts as the center of one $\mathrm{SU}(k)$ factor and also on the $\mathrm{U}(1)$, with $N$ chiral multiplets $\phi_{a}^{i}$ in the $(\mathbf{k}, \mathbf{1})_{\mathbf{1}}$ representation of the gauge group, $N$ chiral multiplets $\tilde{\phi}_{a}^{i}$ in the $(\mathbf{1}, \mathbf{k})_{\mathbf{1}}$ representation of the gauge group, and

$$
\left(\begin{array}{c}
N \\
k
\end{array}\right)
$$

chiral superfields $p_{i_{1} \cdots i_{k}}$ (antisymmetric in their indices) in the $(\mathbf{1}, \mathbf{1})_{-\mathbf{k}}$ representation, with superpotential

$$
W=p_{i_{1} \cdots i_{k}}\left(f^{i_{1} \cdots i_{k}}(B)-\tilde{B}^{i_{1} \cdots i_{k}}\right),
$$

where

$$
B^{i_{1} \cdots i_{k}}=\phi_{a_{1}}^{i_{1}} \cdots \phi_{a_{k}}^{i_{k}} \epsilon^{a_{1} \cdots a_{k}}, \quad \tilde{B}^{i_{1} \cdots i_{k}}=\tilde{\phi}_{a_{1}^{\prime}}^{i_{1}} \cdots \tilde{\phi}_{a_{k}^{\prime}}^{i_{k}} \epsilon^{a_{1}^{\prime} \cdots a_{k}^{\prime}},
$$

are the baryons corresponding to two copies of the Grassmannian, and the $f^{i_{1} \cdots i_{k}}$ (antisymmetric in indices) are linear functions describing the deformation of one copy of the Grassmannian. We claim that, at least for some values of $n$ and $k$, the GLSM above will describe the intersection of the Grassmannians.

As one quick consistency check, it is straightforward to check that the sum of the $\mathrm{U}(1)$ charges will vanish when condition (3.11) is satisfied, so we recover the Calabi-Yau condition as expected.

Let us discuss the discrete quotient in some more detail. Take $\alpha \in \mathrm{U}(1)$ and $g_{1}, g_{2} \in$ $\mathrm{SU}(k)$. Then we have the explicit transformation rules

$$
p_{i_{1} i_{2}} \rightarrow \alpha^{-k} p_{i_{1} i_{2}} \quad \phi^{i} \rightarrow \alpha g_{1} \phi^{i} \quad \tilde{\phi}^{i} \rightarrow \alpha g_{2} \tilde{\phi}^{i}
$$

We see there is a $\mathbb{Z}_{k}$ fixed locus at

$$
\alpha=e^{\frac{2 \pi i}{k} j} \quad g_{1}=g_{2}=e^{-\frac{2 \pi i}{k} j} \mathbf{1}, \quad j=0, \ldots, k-1 .
$$


Modding out by this $\mathbb{Z}_{k}$ action we can rewrite the gauge group as $G=(\mathrm{U}(k) \times \mathrm{U}(k)) / \mathrm{U}(1)$. Explicitly, this is realized by defining $\tilde{g}_{1}=\alpha g_{1} \in \mathrm{U}(k)$ and $\tilde{g}_{2}=\alpha g_{2} \in \mathrm{U}(k)$. Note in particular that $\operatorname{det} \tilde{g}_{1}=\alpha^{k} \operatorname{det} g_{1}=\alpha^{k}=\operatorname{det} \tilde{g}_{2}$, which implies that $p_{i_{1} i_{2}}$ transforms in the inverse determinantal representation of each of the $\mathrm{U}(k) \mathrm{s}$ whereas the baryons transform in the determinantal representation

For practical purposes it is useful to give an explicit parametrization of the maximal torus of the gauge group. We first start off with $\mathrm{U}(1) \times \mathrm{SU}(k) \times \mathrm{SU}(k)$. We denote $\sigma_{0}$ for $\mathrm{U}(1)$ and $\operatorname{diag}\left(\sigma_{h, 1}, \ldots, \sigma_{h, k}\right)(h=1,2)$ for $\mathrm{SU}(k)$, where we have $\sum_{j=1}^{k} \sigma_{h, j}=0$. The elements for the two $\mathrm{U}(k)_{\alpha} \operatorname{are} \operatorname{diag}\left(\sigma_{0}+\sigma_{h, 1}, \ldots, \sigma_{0}+\sigma_{h, k}\right)$. It is convenient to make the following change of coordinates.

$$
\alpha_{1}=\sigma_{0}+\sigma_{1,1} \quad \ldots \quad \alpha_{k}=\sigma_{0}+\sigma_{1, k} \quad \beta_{1}=\sigma_{0}+\sigma_{2,1} \quad \ldots \quad \beta_{k}=\sigma_{0}+\sigma_{2, k}
$$

These coordinates are subject to the condition $\sum_{j=1}^{k} \alpha_{k}=\sum_{j=1}^{j} \beta_{k}$. We will use this to explicitly eliminate one of them. Further note the positive roots of $\mathrm{SU}(k)$ are $\mathbf{e}_{i}-\mathbf{e}_{i+1}$ where the $\mathbf{e}_{j}$ are the basis vectors of $\mathbb{R}^{k}$.

Following [6] we can write down the D-terms associated to the two $\mathrm{U}(k) \mathrm{s}$ :

$$
\begin{array}{ll}
\mathrm{U}(k)_{1}: & \sum_{i=1}^{N} \phi_{a}^{i} \phi^{i \dagger, b}-\sum_{j=1}^{\left(\begin{array}{c}
N \\
k
\end{array}\right)}\left|p_{j}\right|^{2} \delta_{a}^{b}-r \delta_{a}^{b} \\
\mathrm{U}(k)_{2}: & \sum_{i=1}^{N} \tilde{\phi}_{a^{\prime}}^{i} \tilde{\phi}^{i \dagger, b^{\prime}}-\sum_{j=1}^{\left(\begin{array}{c}
N \\
k
\end{array}\right)}\left|p_{j}\right|^{2} \delta_{a^{\prime}}^{b^{\prime}}-r \delta_{a^{\prime}}^{b^{\prime}},
\end{array}
$$

where $r$ is the FI-parameter (for the single overall $\mathrm{U}(1))$ and $j$ combines the $\left(\begin{array}{l}N \\ k\end{array}\right)$ independent index-combinations of the $p$-fields. For $r \gg 0$ this implies that the matrices $\phi \phi^{\dagger}$ and $\tilde{\phi} \tilde{\phi}^{\dagger}$ must have full rank, implying that the $\phi_{a}^{i}$ vevs can be taken to form an orthonormal set of vectors in $\mathbb{C}^{N}[24$, section 4.2$]$, whereas for $r \ll 0$ not all $p$-fields are allowed to vanish simultaneously.

It is also interesting to add and subtract the two sets of D-terms. In the former case one gets

$$
\sum_{i=1}^{N} \phi_{a}^{i} \phi^{i \dagger, b}+\sum_{i=1}^{N} \tilde{\phi}_{a}^{i} \tilde{\phi}^{i \dagger, b}-2 \sum_{k=1}^{\left(\begin{array}{c}
N \\
k
\end{array}\right)}\left|p_{k}\right|^{2} \delta_{a}^{b}-2 r \delta_{a}^{b} .
$$

The diagonal terms correspond to the U(1) D-term. The difference of the two Dterms gives

$$
\sum_{i=1}^{N} \phi_{a}^{i} \phi^{i \dagger, b}-\sum_{i=1}^{N} \tilde{\phi}_{a}^{i} \tilde{\phi}^{i \dagger, b} .
$$

Let us briefly describe the analysis of this model for $r \gg 0$. First, the D terms imply that the $\left\{\phi_{a}^{i}, \tilde{\phi}_{a}^{i}\right\}$ are not all zero, hence the $\left\{B^{i_{1} \cdots i_{k}}, \tilde{B}^{i_{1} \cdots i_{k}}\right\}$ are not all zero. The $\mathrm{F}$ terms imply

$$
\tilde{B}^{i_{1} \cdots i_{k}}=f^{i_{1} \cdots i_{k}}(B), \quad p_{i_{1} \cdots i_{k}} \frac{\partial}{\partial \phi_{a}^{i}} f^{i_{1} \cdots i_{k}}(B)=0=p_{i_{1} \cdots i_{k}} \frac{\partial}{\partial \tilde{\phi}_{a}^{i}} \tilde{B}^{i_{1} \cdots i_{k}} .
$$


The first $\mathrm{F}$ term equation clearly restricts to the intersection, but we still need to dispense with the $p$ 's.

Next, note that from the D-terms, since the $\left\{\phi_{a}^{i}\right\}$ and $\left\{\tilde{\phi}_{a^{\prime}}^{i}\right\}$ vevs each form a set of $k$ linearly independent vectors in $\mathbb{C}^{N}$, the dual quantities

$$
\epsilon^{a_{1} \cdots a_{k}} \phi_{a_{2}}^{i_{2}} \cdots \phi_{a_{k}}^{i_{k}}, \quad \epsilon^{a_{1}^{\prime} \cdots a_{k}^{\prime}} \tilde{\phi}_{a_{2}^{\prime}}^{i_{2}} \cdots \tilde{\phi}_{a_{k}^{\prime}}^{i_{k}}
$$

also form two sets of $k$ linearly independent vectors (in a larger vector space), hence the derivatives

$$
\frac{\partial}{\partial \phi_{a}^{i}} B^{i_{1} \cdots i_{k}}, \quad \frac{\partial}{\partial \tilde{\phi}_{a^{\prime}}^{i}} \tilde{B}^{i_{1} \cdots i_{k}}
$$

also form two sets of linearly independent vectors under certain genericity conditions [6]. For generic rotations $f^{i_{1} \cdots i_{k}}$,

$$
\frac{\partial}{\partial \phi_{a}^{i}} f^{i_{1} \cdots i_{k}}(B)=f_{j_{1} \cdots j_{k}}^{i_{1} \cdots i_{k}} \frac{\partial}{\partial \phi_{a}^{i}} B^{j_{1} \cdots j_{k}}
$$

form a set of $\left(\begin{array}{l}N \\ k\end{array}\right)$ linearly independent vectors, and so the $\mathrm{F}$ term equations force all the $p_{i_{1} \cdots i_{k}}$ to vanish. However, for nongeneric choices of $f$, not all the resulting vectors will be linearly independent, and so one can get noncompact branches of unconstrained $p$ 's, indicating a physical singularity. This is in agreement with the smoothness criteria in the mathematics literature.

To make this more concrete, let us consider the special case that $k=2$. In this case, $\partial B / \partial \phi \propto \phi_{a}^{i}$. From the D-terms, the $\phi_{a}^{i}$ define two linearly independent vectors in $\mathbb{C}^{N}$, and the $\tilde{\phi}_{a^{\prime}}^{i}$ denote another two linearly independent vectors in $\mathbb{C}^{N}$. For generic rotations, the F-term constraints include

$$
p_{i_{1} i_{2}} \epsilon^{a b} f_{j_{1} j_{2}}^{i_{1} i_{2}} \phi_{b}^{j_{2}}=0=p_{i_{1} i_{2}} \epsilon^{a^{\prime} b^{\prime}} \tilde{\phi}_{b^{\prime}}^{i_{2}} .
$$

Generic rotation matrices $f_{j_{1} j_{2}}^{i_{1} i_{2}}$ can rotate the $\phi_{a}^{i}$ into $\left(\begin{array}{c}N \\ 2\end{array}\right)$ linearly independent vectors, and in such cases the only way to satisfy the F-term constraints is for all $p_{i_{1} i_{2}}=0$.

However, for nongeneric rotations $f$, we can have noncompact branches. Consider for example the case of trivial rotations (again for $k=2$ ), in which $f$ is the identity. Identify $\phi_{a}^{i}=\tilde{\phi}_{a^{\prime}}^{i}$, then omitting the now-irrelevant $\epsilon^{a b}$, the F-term constraints become

$$
p_{i_{1} i_{2}} \phi_{a}^{i_{2}}=0 \text {. }
$$

Suppose for example we are at a point where $\phi_{a}^{i} \propto \delta_{a}^{i}$, which is consistent with D-terms. Then the $p_{i j}$ for $i, j>2$ are completely unconstrained, and their vevs can run along a noncompact branch. This is consistent with the mathematics result mentioned earlier that for the trivial rotation, the self-intersection of $G(k, N)$ is singular as an element of the family.

Now that we have described general aspects of these GLSMs, let us look at the result for a particular model. For example, consider $G(2,5)$. Here,

$$
\begin{aligned}
2 N k & =20, \\
\left(\begin{array}{c}
N \\
k
\end{array}\right) & =10,
\end{aligned}
$$


hence for generic maps $f^{i_{1} \cdots i_{k}}$, all the $p$ 's will be forced to vanish, and this GLSM will correctly describe the corresponding geometry. However, for nongeneric $f^{i_{1} \cdots i_{k}}$, this description will break down. For example, if $f^{i_{1} \cdots i_{k}}$ is the identity, then instead of $2 N k$ independent equations, there are only $N k=10$ independent equations, at which point linear algebra suggests the $p_{i_{1} \cdots i_{k}}$ may become nonzero, and our description of the geometry breaks down.

This description of the geometry meshes with the mathematics of the intersection of $G(2,5)$ with itself, which is Calabi-Yau.

In the Calabi-Yau case, we can use the GLSM to extract further characteristic information about the Kähler moduli space. The singularities in the Kähler moduli space coincide with those loci in the FI-theta parameter space, where Coulomb branches or mixed Coulomb-Higgs branches emerge. Since our examples are one-parameter models, there are no mixed Coulomb-Higgs branches and the singularities are determined by computing the critical set of the effective potential on the Coulomb branch. This is given by

$$
\widetilde{W}_{\mathrm{eff}}=-t(\sigma)-\sum_{i} Q_{i}(\sigma)\left(\log \left(Q_{i}(\sigma)\right)-1\right)+\pi i \sum_{\rho>0} \rho(\sigma),
$$

where $t=r-i \theta$ is the FI-theta parameter, $Q_{i}$ are the gauge charges of the matter fields, $\sigma$ parametrizes the maximal torus of the gauge group and $\rho>0$ are the positive roots. For our examples this reads

$$
\begin{aligned}
\widetilde{\mathcal{W}}_{\mathrm{eff}}= & -t \sum_{j=1}^{k} \alpha_{k}+\pi i \sum_{j=1}^{k-1}\left[\left(\alpha_{j}-\alpha_{j+1}\right)+\left(\beta_{j}-\beta_{j+1}\right)\right] \\
& -\left(\begin{array}{c}
N \\
k
\end{array}\right) \sum_{j=1}^{k}\left(-\alpha_{j}\right)\left(\log \left(\sum_{j=1}^{k}\left(-\alpha_{j}\right)\right)-1\right) \\
& -N \sum_{j=1}^{k}\left[\alpha_{j}\left(\log \alpha_{j}-1\right)+\beta_{j}\left(\log \beta_{j}-1\right)\right],
\end{aligned}
$$

where we have used the parametrization of the maximal torus, given above. The critical set of $\widetilde{W}_{\text {eff }}$ is given by the following $2 k-1$ equations:

$$
\begin{aligned}
e^{-t} & =(-1)^{j-1} \frac{\alpha_{j}^{N}\left(\alpha_{1}+\ldots+\alpha_{k}-\beta_{1}-\ldots-\beta_{k-1}\right)^{N}}{\left(\sum_{k=1}^{k}\left(-\alpha_{j}\right)\right)^{\left(\begin{array}{c}
N \\
k
\end{array}\right)},} \quad j=1, \ldots, k, \\
1 & =(-1)^{j-1} \frac{\left(\alpha_{1}+\ldots+\alpha_{k}-\beta_{1}-\ldots-\beta_{k-1}\right)^{N}}{\beta_{j}^{N}} \quad j=1, \ldots, k-1 .
\end{aligned}
$$

Here we have eliminated $\beta_{k}$ and have used (3.11) to simplify the expressions. Solving these equations gives the points $e^{-t}$ where the Coulomb branches are located. In section 4 we will discuss the case $k=2, N=5$ in more detail.

\subsubsection{Intersection with Veronese embedding}

So far we have discussed the intersection of a single Grassmannian $G(k, N)$ with its own Plücker image after a linear deformation. In principle, we can apply the same ideas to 
intersections of different Grassmannians. Their Plücker embeddings will land in projective spaces of different sizes, so one must specify a map between projective spaces, but given such a map, the rest is straightforward.

Specifically, if we compose the Plücker embedding of a Grassmannian $G(k, N)$ with a degree $d$ Veronese map, the result lies in the same projective space as the Plücker embedding of the Grassmannian $G\left(k^{\prime}, N^{\prime}\right)$ for

$$
k^{\prime}=d, \quad N^{\prime}=\left(\begin{array}{c}
N \\
k
\end{array}\right)-1+d .
$$

The intersection can be described by the GLSM with gauge group

$$
\frac{\mathrm{U}(1) \times \mathrm{SU}(k) \times \mathrm{SU}\left(k^{\prime}\right)}{\mathbb{Z}_{k}},
$$

with matter

- $N$ chiral superfields in the $(\mathbf{k}, \mathbf{1})_{1}$ representation,

- $N^{\prime}$ chiral superfields in the $\left(\mathbf{1}, \mathbf{k}^{\prime}\right)_{k}$ representation,

- $\left(\begin{array}{c}N^{\prime} \\ k^{\prime}\end{array}\right)$ chiral superfields $p_{i_{1}^{\prime} \cdots i_{k^{\prime}}^{\prime}}$ (antisymmetric in their indices) in the $(\mathbf{1}, \mathbf{1})_{-k k^{\prime}}$ representation,

and superpotential

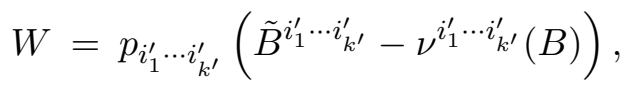

with baryons $B^{i_{1} \cdots i_{k}}, \tilde{B}^{i_{1}^{\prime} \cdots i_{k^{\prime}}^{\prime}}$ constructed from $\mathrm{SU}(k)$ - and $\mathrm{SU}\left(k^{\prime}\right)$-invariant combinations of fundamental-charged chiral superfields, as usual.

In passing, the reader should note that the finite group quotients are such that the Grassmannian input to the Veronese embedding is built with a $\mathrm{U}(k)$ gauge group, but the Grassmannian intersected at the output is constructed with a $\mathrm{U}(1) \times \mathrm{SU}\left(k^{\prime}\right)$ gauge group. This is closely related to the concern with nonminimally-charged chiral superfields corresponding to homogeneous coordinates in the target of the Veronese embedding. First, since the Lie algebras are the same in both cases, the perturbative physics is identical; these differences in finite group quotients can only affect nonperturbative sectors. Having gauge group $\mathrm{U}(1) \times \mathrm{SU}\left(k^{\prime}\right)$ reflects the fact that the only allowed maps into the Grassmannian have degree divisible by $k^{\prime}$, the degree of the Veronese embedding, as discussed in other circumstances in e.g. [20-23].

Judging from the $\mathrm{U}(1)$ charges, the Calabi-Yau condition is that

$$
N+N^{\prime} k^{\prime}=\left(\begin{array}{c}
N^{\prime} \\
k^{\prime}
\end{array}\right) k^{\prime} .
$$

We can see this mathematically as follows. Let $G=G(k, N), G^{\prime}=G\left(k^{\prime}, N^{\prime}\right)$, and $\mathbb{P}^{\prime}$ denotes the projective space of dimension

$$
\left(\begin{array}{c}
N^{\prime} \\
k^{\prime}
\end{array}\right)-1
$$


From the adjunction formula,

$$
K_{G^{\prime}}=\left.K_{\mathbb{P}^{\prime}}\right|_{G^{\prime}}+c_{1}\left(N_{G^{\prime} / \mathbb{P}^{\prime}}\right),
$$

and if the intersection $X=G \cap G^{\prime}$ is transversal, then we have $\left.N_{X / G} \cong \nu^{*} N_{G^{\prime} / \mathbb{P}^{\prime}}\right|_{X}$, hence

$$
c_{1}\left(N_{X / G}\right)=d c_{1}\left(\left.N_{G^{\prime} / \mathbb{P}^{\prime}}\right|_{X}\right),
$$

where $\nu$ denotes the degree $d$ Veronese embedding. Using the adjunction formula again, we have

$$
\begin{aligned}
K_{X} & =\left.K_{G}\right|_{X}+c_{1}\left(N_{X / G}\right), \\
& =\left.K_{G}\right|_{X}+d\left[\left.K_{G^{\prime}}\right|_{X}-\left.K_{\mathbb{P}^{\prime}}\right|_{X}\right], \\
& =N+d\left[N^{\prime}-\left(\begin{array}{c}
N^{\prime} \\
k^{\prime}
\end{array}\right)\right],
\end{aligned}
$$

and so we recover the Calabi-Yau condition (3.40).

The expected dimension of this intersection is

$$
k^{\prime}\left(N^{\prime}-k^{\prime}\right)+k(N-k)-\left(\begin{array}{c}
N^{\prime} \\
k^{\prime}
\end{array}\right)+1 .
$$

This can be derived from the fact that the expected dimension of $X \cap Y \subset S$ is

$$
\operatorname{dim} X+\operatorname{dim} Y-\operatorname{dim} S .
$$

As a result, nontrivial examples are limited in number. One class of examples is the case $d=1$, which describe the intersection of the Grassmannian $G(k, N)$ with the projective space that defines the image of its Plücker embedding. In other words, the case $d=1$ corresponds to a complicated description of the Grassmannian $G(k, N)$. We can see this directly in physics as follows. From our general prescription, the GLSM is a

$$
\frac{\mathrm{U}(1) \times \mathrm{SU}(k) \times \mathrm{SU}(1)}{\mathbb{Z}_{k}}=\mathrm{U}(k)
$$

gauge theory with matter

- $N$ chiral superfields $\phi_{a}^{i}$ in the fundamental representation,

- $N^{\prime}=\left(\begin{array}{l}N \\ k\end{array}\right)$ chiral superfields $\tilde{\phi}^{i^{\prime}}=\tilde{\phi}^{i_{1} \cdots i_{k}}$ in the $\mathbf{1}_{k}$ representation,

- $N^{\prime}$ chiral superfields $p_{i^{\prime}}=p_{i_{1} \cdots i_{k}}$ in the $\mathbf{1}_{-k}$ representation,

and superpotential

$$
W=p_{i_{1} \cdots i_{k}}\left(\tilde{\phi}^{i_{1} \cdots i_{k}}-B^{i_{1} \cdots i_{k}}\right) .
$$

Integrating out the $p$ 's and $\tilde{\phi}$ 's immediately recovers the Grassmannian $G(k, N)$.

Another class of examples has $d=2$ and $k=1$. In these examples, one intersects the Plücker embedding of the Grassmannian $G(2, N+1)$ with the Veronese image of $G(1, N)=$ $\mathbb{P}^{N-1}$. For $N=2,3$, these have expected dimension 1 . 


\subsubsection{Intersection with Segre embedding}

For another example, suppose we have three Grassmannians $G\left(k_{1}, N_{1}\right), G\left(k_{2}, N_{2}\right)$, $G\left(k_{3}, N_{3}\right)$ where the $k$ 's and $N$ 's obey

$$
\left(\begin{array}{l}
N_{3} \\
k_{3}
\end{array}\right)=\left(\begin{array}{l}
N_{1} \\
k_{1}
\end{array}\right)\left(\begin{array}{c}
N_{2} \\
k_{2}
\end{array}\right) .
$$

Consider the intersection of the Plücker embedding of $G\left(k_{3}, N_{3}\right)$ with the Segre embedding of the images of the Plücker embeddings of $G\left(k_{1}, N_{1}\right)$ and $G\left(k_{2}, N_{2}\right)$. We can realize this in a GLSM as follows. Consider the GLSM with gauge group

$$
\mathrm{U}\left(k_{1}\right) \times \mathrm{U}\left(k_{2}\right) \times \mathrm{SU}\left(k_{3}\right),
$$

with matter

- $N_{1}$ chiral multiplets in representation $\left(\mathbf{k}_{\mathbf{1}}, \mathbf{1}, \mathbf{1}\right)$,

- $N_{2}$ chiral multiplets in representation $\left(\mathbf{1}, \mathbf{k}_{\mathbf{2}}, \mathbf{1}\right)$,

- $N_{3}$ chiral multiplets in the fundamental of $\mathrm{SU}\left(k_{3}\right)$, of charge $k_{1}$ under $\operatorname{det} \mathrm{U}\left(k_{1}\right)$ and charge $k_{2}$ under det $\mathrm{U}\left(k_{2}\right)$,

- $\left(\begin{array}{l}N_{3} \\ k_{3}\end{array}\right)$ chiral multiplets $p_{i_{1} \cdots i_{k 3}}$ of charge $-k_{1}$ under det $\mathrm{U}\left(k_{1}\right)$ and charge $-k_{2}$ under $\operatorname{det} \mathrm{U}\left(k_{2}\right)$,

and superpotential

$$
W=p_{i_{1} \cdots i_{k 3}}\left(B_{3}^{i_{1} \cdots i_{k 3}}-S^{i_{1} \cdots i_{k 3}}\left(B_{1}, B_{2}\right)\right),
$$

where $B_{i}$ denotes baryons for $\mathrm{SU}\left(k_{i}\right)$ (i.e. the Plücker images of each Grassmannian), and $S^{i_{1} \cdots i_{k 3}}$ denotes the image of the Segre embedding map.

In passing, the fact that there is no $\mathbb{Z}_{k_{3}}$ finite group quotient in the gauge group reflects the fact that maps into the third Grassmannian are of degree satisfying certain divisibility conditions, as we have seen previously in discussions of GLSM realizations of Veronese embeddings.

It is straightforward to find (not necessarily Calabi-Yau) examples, from identities such as

$$
\left(\begin{array}{l}
6 \\
1
\end{array}\right)\left(\begin{array}{l}
6 \\
3
\end{array}\right)=\left(\begin{array}{c}
10 \\
3
\end{array}\right) \text {. }
$$

It is straightforward to check from $\mathrm{U}(1)$ charges that the Calabi-Yau condition is

$$
N_{1}+N_{3}=\left(\begin{array}{c}
N_{3} \\
k_{3}
\end{array}\right), \quad N_{2}+N_{3}=\left(\begin{array}{c}
N_{3} \\
k_{3}
\end{array}\right) .
$$

We can also derive this mathematically as follows. Let $X=G\left(k_{1}, N_{1}\right) \times G\left(k_{2}, N_{2}\right)$, $Y=G\left(k_{3}, N_{3}\right), S$ the projective space in which $Y$ is embedded via Plücker, and $W$ the intersection. The Calabi-Yau condition can then be stated as

$$
\left.K_{X}\right|_{W}+\left.K_{Y}\right|_{X}=\left.K_{S}\right|_{W}
$$


In the present case,

$$
K_{X}=\left(-N_{1},-N_{2}\right), \quad K_{Y}=-N_{3} .
$$

The Calabi-Yau condition then becomes the statement derived above from U(1) charges.

\subsection{Intersections of Pfaffians}

In this section, we will describe perturbative GLSMs for intersections of Pfaffians, following the PAX and PAXY models described in [9].

\subsubsection{PAX models}

First, let us recall the PAX model of a GLSM for a Pfaffian. Suppose the Pfaffian variety we wish to describe arises as the locus $\{\operatorname{rank} A(\phi) \leq k\} \subset \mathbb{P}^{n}$, for $A(\phi)$ an $N \times N$ matrix whose entries are homogeneous in the homogeneous coordinates of a projective space $\mathbb{P}^{n}$. For simplicity, let us assume that each entry in the matrix $A$ has degree $d$.

In the PAX model, a GLSM describing this variety is given as a $\mathrm{U}(1) \times \mathrm{U}(N-k)$ gauge theory with the following matter:

- $n+1$ chiral superfields $\phi_{i}$, of charge 1 under the $\mathrm{U}(1)$ and neutral under $\mathrm{U}(N-k)$ (corresponding to homogeneous coordinates on $\mathbb{P}^{n}$ ),

- $N$ chiral superfields $x_{i a}$ transforming in the $\overline{\mathbf{N}-\mathbf{k}}$ representation of $\mathrm{U}(N-k)$ (and neutral under the $\mathrm{U}(1))$,

- and $N$ chiral superfields $p_{i}^{a}$, transforming in the $\mathbf{N}-\mathbf{k}$ representation of $\mathrm{U}(N-k)$ and of charge $-d$ under the $\mathrm{U}(1)$,

with superpotential

$$
W=\sum_{i=1}^{N} \sum_{a=1}^{N-k} p_{i}^{a} A^{i j}(\phi) x_{j a} .
$$

(The name of the model follows from the form of the superpotential.)

We could describe the intersection of two loci as follows. Suppose we have an $N_{1} \times N_{1}$ matrix $A^{i j}(\phi)$, entries homogeneous of degree $d_{1}$ in the homogeneous coordinates on $\mathbb{P}^{n}$, and an $N_{2} \times N_{2}$ matrix $B^{i j}(\phi)$, entries homogeneous of degree $d_{2}$ in the homogeneous coordinates on the same $\mathbb{P}^{n}$, and we want to describe the intersection

$$
\left\{\operatorname{rank} A \leq k_{1}\right\} \cap\left\{\operatorname{rank} B \leq k_{2}\right\} \subset \mathbb{P}^{n} .
$$

To do this, we will use two copies of the PAX model. Specifically, consider the GLSM defined by a $\mathrm{U}(1) \times \mathrm{U}\left(N_{1}-k_{1}\right) \times \mathrm{U}\left(N_{2}-k_{2}\right)$ gauge theory with the following matter:

- $n+1$ chiral superfields $\phi_{i}$ of charge 1 under the $\mathrm{U}(1)$ and neutral under $\mathrm{U}\left(N_{1}-k_{1}\right) \times$ $\mathrm{U}\left(N_{2}-k_{2}\right)$ (corresponding to homogeneous coordinates on $\left.\mathbb{P}^{n}\right)$,

- $n+1$ chiral superfields $\tilde{\phi}_{i}$ of charge 1 under the $\mathrm{U}(1)$ and neutral under $\mathrm{U}\left(N_{1}-k_{1}\right) \times$ $\mathrm{U}\left(N_{2}-k_{2}\right)$, corresponding to homogeneous coordinates on a second copy of $\mathbb{P}^{n}$, 
- $n+1$ chiral superfields $q_{i}$ of charge -1 under the $\mathrm{U}(1)$ and neutral under $\mathrm{U}\left(N_{1}-k_{1}\right) \times$ $\mathrm{U}\left(N_{2}-k_{2}\right)$, multiplying superpotential terms identifying the two sets of homogeneous coordinates,

- $N_{1}$ chiral superfields $x_{i a}$ transforming in the $\left(\overline{\mathbf{N}_{\mathbf{1}}-\mathbf{k}_{\mathbf{1}}}, \mathbf{1}\right)$ representation of $\mathrm{U}\left(N_{1}-\right.$ $\left.k_{1}\right) \times \mathrm{U}\left(N_{2}-k_{2}\right)$ (and neutral under the $\mathrm{U}(1)$ ),

- $N_{1}$ chiral superfields $p_{i}^{a}$, transforming in the $\left.\left(\mathbf{N}_{\mathbf{1}}-\mathbf{k}_{\mathbf{1}}\right), \mathbf{1}\right)$ representation of $\mathrm{U}\left(N_{1}-\right.$ $\left.k_{1}\right) \times \mathrm{U}\left(N_{2}-k_{2}\right)$ and of charge $-d_{1}$ under the $\mathrm{U}(1)$,

- $N_{2}$ chiral superfields $\tilde{x}_{i^{\prime} a^{\prime}}$ transforming in the $\left(\mathbf{1}, \overline{\mathbf{N}_{\mathbf{2}}-\mathbf{k}_{\mathbf{2}}}\right)$ representation of $\mathrm{U}\left(N_{1}-\right.$ $\left.k_{1}\right) \times \mathrm{U}\left(N_{2}-k_{2}\right)$ (and neutral under the $\mathrm{U}(1)$ ),

- $N_{2}$ chiral superfields $\tilde{p}_{i^{\prime}}^{a^{\prime}}$, transforming in the $\left(\mathbf{1}, \mathbf{N}_{\mathbf{2}}-\mathbf{k}_{\mathbf{2}}\right)$ representation of $\mathrm{U}\left(N_{1}-\right.$ $\left.k_{1}\right) \times \mathrm{U}\left(N_{2}-k_{2}\right)$ and of charge $-d_{2}$ under the $\mathrm{U}(1)$,

with superpotential

$$
W=\sum_{i=1}^{N_{1}} \sum_{a=1}^{N_{1}-k_{1}} p_{i}^{a} A^{i j}(\phi) x_{j a}+\sum_{i^{\prime}=1}^{N_{2}} \sum_{a^{\prime}=1}^{N_{2}-k_{2}} \tilde{p}_{i^{\prime}}^{a^{\prime}} B^{i^{\prime} j^{\prime}}(\tilde{\phi}) \tilde{x}_{j^{\prime} a^{\prime}}+\sum_{i=1}^{n+1} q_{i}\left(\phi_{i}-\tilde{\phi}_{i}\right) .
$$

Next, for simplicity, we will assume that the $q_{i}$ have been integrated out, so that we can identify $\phi_{i}=\tilde{\phi}_{i}$.

Now, let us carefully analyze this theory, closely following [9, section 3.2]. We shall focus on D- and F-terms for the extra data above the base toric variety, so for example we assume FI parameters are such that not all the $\phi$ vanish. D-terms for $\mathrm{U}\left(N_{1}-k_{1}\right)$, $\mathrm{U}\left(N_{2}-k_{2}\right)$ imply

$$
p_{i}^{a}\left(p^{\dagger}\right)_{b}^{i}-\left(x^{\dagger}\right)^{i a} x_{i b}=r_{1} \delta_{b}^{a}, \quad \tilde{p}_{i}^{a^{\prime}}\left(\tilde{p}^{\dagger}\right)_{b^{\prime}}^{i}-\left(\tilde{x}^{\dagger}\right)^{i a^{\prime}} \tilde{x}_{i b^{\prime}}=r_{2} \delta_{b^{\prime}}^{a^{\prime}}
$$

Define $E_{a}^{i}(\phi, x)=A(\phi)^{i j} x_{j a}, \tilde{E}_{a^{\prime}}^{i^{\prime}}=B(\phi)^{i^{\prime} j^{\prime}} \tilde{x}_{j^{\prime} a^{\prime}}$. The F-term conditions can then be expressed as

$$
\begin{array}{r}
A(\phi)^{i j} x_{j a}=0, \quad \tilde{E}_{a^{\prime}}^{i^{\prime}}=B(\phi)^{i^{\prime} j^{\prime}} \tilde{x}_{j^{\prime} a^{\prime}}=0, \\
p_{i}^{a} \frac{\partial E_{a}^{i}}{\partial(\phi, x, \tilde{x})}+\tilde{p}_{i^{\prime}}^{a^{\prime}} \frac{\partial \tilde{E}_{a^{\prime}}^{i^{\prime}}}{\partial(\phi, x, \tilde{x})}=0 .
\end{array}
$$

The first two conditions demonstrate that the vacua lie along the intersection

$$
\{(\phi, x) \mid A(\phi) x=0\} \cap\{(\phi, \tilde{x}) \mid B(\phi) \tilde{x}=0\} .
$$

Each factor is a (resolution of) a Pfaffian describing the locus where the rank of a matrix ( $A$ or $B$ ) is bounded by $k$. Following standard methods, the derivatives $\partial E, \partial \tilde{E}$ each span the normal bundle to each factor. The set of such is linearly independent if the intersection is smooth, and so we see from the last set of F-term conditions that smoothness implies that $p=\tilde{p}=0$. 


\subsubsection{PAXY models}

Let us now consider analogous constructions involving the PAXY models [9], which will play an important role in understanding dual theories later in this paper.

The PAXY model that describes the locus on which an $n \times n$ matrix $A(\phi)$ can be factored into a product $Y X$ of two matrices (one $n \times k$, the other $k \times n$ ) can be described by a GLSM with gauge group $\mathrm{U}(k)$ and matter as follows:

- chiral superfields $\Phi$, charged under a different gauge factor, describing the space in which the Pfaffian lives,

- $n$ chiral superfields in the fundamental of $\mathrm{U}(k)$, defining a $k \times n$ matrix $X$,

- $n$ chiral superfields in the antifundamental of $\mathrm{U}(k)$, defining a $n \times k$ matrix $Y$,

- $n^{2}$ chiral superfields forming an $n \times n$ matrix $P$,

and superpotential

$$
W=\sum_{i, j=1}^{n} P_{j i}\left(A(\Phi)_{i j}-\sum_{a=1}^{k} Y_{i a} X_{a j}\right)
$$

To describe the intersection of two closely analogous Pfaffians, defined by matrices $A(\Phi), B(\Phi)$, each with the same factorization constraint, we could use a $\mathrm{U}(k) \times \mathrm{U}(k)$ gauge theory with matter

- chiral superfields $\Phi$, charged under a different gauge factor, describing the space in which the intersection of Pfaffians lives,

- two sets of $n$ chiral superfields, each set in the fundamental of a $\mathrm{U}(k)$ factor, defining $k \times n$ matrices $X, \tilde{X}$

- two sets of $n$ chiral superfields, each in the antifundamental of a $\mathrm{U}(k)$ factor, defining $n \times k$ matrices $Y, \tilde{Y}$,

- two sets of $n^{2}$ chiral superfields forming two $n \times n$ matrices $P, \tilde{P}$,

and superpotential

$$
W=\sum_{i, j=1}^{n} P_{j i}\left(A(\Phi)_{i j}-\sum_{a=1}^{k} Y_{i a} X_{a j}\right)+\sum_{i, j=1}^{n} \tilde{P}_{j i}\left(B(\Phi)_{i j}-\sum_{a^{\prime}=1}^{k} \tilde{Y}_{i a^{\prime}} \tilde{X}_{a^{\prime} j}\right)
$$

Let us work systematically through the analysis. (We will closely follow [9, section 3.3].) We assume that there is a phase in which D terms imply that the $\Phi$ 's are not all zero, and work in that phase. The D-term constraint for the $\mathrm{U}(k)$ is

$$
X_{a i} X_{i b}^{\dagger}-Y_{a i}^{\dagger} Y_{i b}=r \delta_{a b}, \quad \tilde{X}_{a^{\prime} i} \tilde{X}_{i b^{\prime}}^{\dagger}-\tilde{Y}_{a^{\prime} i}^{\dagger} \tilde{Y}_{i b^{\prime}}=\tilde{r} \delta_{a^{\prime} b^{\prime}}
$$


Assuming that $r \gg 0, \tilde{r} \gg 0$, this forces $X$ and $\tilde{X}$ to have rank $k$. The F-term constraints have the form

$$
\begin{array}{rlrl}
A(\Phi)_{i j}=\sum_{a=1}^{k} Y_{i a} X_{a j}, & B(\Phi)_{i j} & =\sum_{a^{\prime}=1}^{k} \tilde{Y}_{i a^{\prime}} \tilde{X}_{a^{\prime} j}, & \\
P_{j i} Y_{i a}=0, & P_{j i} X_{a j} & =0, & \\
P_{j i} \frac{\partial A(\Phi)_{i j}}{\partial \Phi_{\alpha}}+\tilde{P}_{j i} \frac{\partial B(\Phi)_{i j}}{\partial \Phi_{\alpha}} & =0 . &
\end{array}
$$

With the exception of the last constraint, the F-term constraints above are identical to those appearing in two copies of the PAXY model, as analyzed in [9, section 3.3]. It will be helpful to define

$$
E_{i j}=A(\Phi)_{i j}-\sum_{a=1}^{k} Y_{i a} X_{a j}, \quad \tilde{E}_{i j}=B(\Phi)_{i j}-\sum_{a^{\prime}=1}^{k} \tilde{Y}_{i a^{\prime}} \tilde{X}_{a^{\prime} j}
$$

so that the remaining F-term conditions, beyond $E=0=\tilde{E}$, can all be written as

$$
P_{j i} \frac{\partial E(\Phi, X, Y, \tilde{X}, \tilde{Y})_{i j}}{\partial(\Phi, X, Y, \tilde{X}, \tilde{Y})}+\tilde{P}_{j i} \frac{\partial \tilde{E}(\Phi, \tilde{X}, \tilde{Y})_{i j}}{\partial(\Phi, X, Y, \tilde{X}, \tilde{Y})}=0
$$

As in the discussion of the PAX model, the derivatives $\partial E, \partial \tilde{E}$ span the normal bundle. These will be linearly independent if the intersection is smooth, so we see from the last set of F-term conditions that smoothness implies that $P=0=\tilde{P}$.

\subsubsection{PAXY for antisymmetric matrices}

Later in this paper we'll see examples of intersections of Pfaffians for antisymmetric matrices. Suppose we have $N \times N$ antisymmetric matrices $A, B$, each with entries linear in the homogeneous coordinates of $\mathbb{P}^{n}$, and we want to compute the intersection of Pfaffians defined by the rank $k$ locus of each matrix. We assume $N$ is odd, and then of necessity $k$ is even.

The GLSM describing the Pfaffian for a single antisymmetric matrix $A$, of the form above, following [9, section 3.5], is defined by a

$$
\frac{\mathrm{U}(1) \times \mathrm{USp}(k)}{\mathbb{Z}_{2}}
$$

gauge theory with matter

- $n+1$ chiral superfields $\phi$, forming the homogeneous coordinates on the projective space, of charge 2 under the $\mathrm{U}(1)$ and neutral under $\operatorname{USp}(k)$,

- $N$ chiral superfields $x_{i a}$ in the fundamental of $\operatorname{USp}(k)$, and of charge 1 under the $\mathrm{U}(1)$,

- $N^{2}$ chiral superfields $p_{i j}$ of charge -2 under the U(1) and neutral under $\operatorname{USp}(k)$, 
with superpotential

$$
W=\sum_{i j} p_{i j}\left(A(\phi)_{i j}-x_{i a} x_{j b} J^{a b}\right)
$$

where $J$ is the $k \times k$ antisymmetric matrix in block form

$$
J=-\left[\begin{array}{cc}
0 & 1 \\
-1 & 0
\end{array}\right] .
$$

With that in mind, it should be clear that the intersection of two such Pfaffians, defined by antisymmetric $N \times N$ matrices $A, B$, each linear in the homogeneous coordinates of $\mathbb{P}^{n}$, is defined by a

$$
\frac{\mathrm{U}(1) \times \mathrm{USp}(k) \times \mathrm{USp}(k)}{\mathbb{Z}_{2} \times \mathbb{Z}_{2}}
$$

gauge theory with matter

- $n+1$ chiral superfields $\phi$, forming the homogeneous coordinates on the projective space, in representation $(\mathbf{1}, \mathbf{1})_{2}$ of the gauge group,

- $N$ chiral superfields $x_{i a}$ in the representation $(\mathbf{k}, \mathbf{1})_{1}$ of the gauge group,

- $N$ chiral superfields $\tilde{x}_{i^{\prime} a^{\prime}}$ in the representation $(\mathbf{1}, \mathbf{k})_{1}$ of the gauge group,

- $N^{2}$ chiral superfields $p_{i j}$ in the representation $(\mathbf{1}, \mathbf{1})_{-2}$,

- $N^{2}$ chiral superfields $\tilde{p}_{i^{\prime} j^{\prime}}$ in the representation $(\mathbf{1}, \mathbf{1})_{-2}$,

with superpotential

$$
W=\sum_{i j} p_{i j}\left(A(\phi)_{i j}-x_{i a} x_{j b} J^{a b}\right)+\sum_{i^{\prime} j^{\prime}} \tilde{p}_{i^{\prime} j^{\prime}}\left(B(\phi)_{i^{\prime} j^{\prime}}-\tilde{x}_{i^{\prime} a^{\prime}} \tilde{x}_{j^{\prime} b^{\prime}} J^{a^{\prime} b^{\prime}}\right) .
$$

It is straightforward to check that the sum of the $\mathrm{U}(1)$ charges will vanish when

$$
n+1=N(N-k) \text {. }
$$

We can derive this Calabi-Yau condition mathematically as follows. First, consider an $N \times N$ skew-symmetric matrix, $N$ odd, of generic variables. There are

$$
M=\left(\begin{array}{c}
N \\
2
\end{array}\right)
$$

such variables. Let $X$ denote the locus in $\mathbb{P} \equiv \mathbb{P}^{M-1}$ where the rank of this matrix is less than $k$. The structure sheaf $\mathcal{O}_{X}$ admits a resolution of the form [25, theorem 6.4.1(c)]

$$
0 \longrightarrow \mathcal{O}_{\mathbb{P}}(-N(N-k) / 2) \longrightarrow \cdots \longrightarrow \mathcal{O}_{\mathbb{P}}
$$

If we specialize the generic variables to linear forms in $\mathbb{P}^{n}$, then the resolution remains exact if and only if the codimension is preserved. In that case, the resolution above stays the same, so by adjunction, the canonical bundle of the Pfaffian variety $X$ is $\mathcal{O}_{X}(N(N-k) / 2-n-1)$. 
Now, for the intersection of two such Pfaffians, call them $X$ and $Y$, to be Calabi-Yau, one must require

$$
K_{X}+K_{Y}=K_{\mathbb{P}}
$$

or explicitly

$$
2(N(N-k)-n-1)=-(n+1),
$$

hence $N(N-k)=n+1$, the condition derived above from $\mathrm{U}(1)$ charges.

\section{Phases of GLSMs for self-intersection of $G(2, N)$ for $N$ odd}

In this section we will study the GLSM for the self-intersection of two copies of $G(2, N)$, one linearly rotated. We previously discussed this setup in section 3.2.1.

First, let us recall the condition (3.10) for such self-intersections to be nonempty. In the present case of $G(2, N)$ for $N$ odd, it is straightforward to check that the only nonempty cases are those for which $N \leq 7$. Furthermore, from the expected dimension formula (3.9), the case $N=3$ has dimension 2 , the case $N=5$ has dimension 3 , and the case $N=7$ has dimension 0 .

Of these three cases, the case in which we are primarily interested is the case $N=5$. This case corresponds to a Calabi-Yau threefold: from equation (3.11), we see that this intersection is be Calabi-Yau, and for generic deformations, the formula for the expected dimension (3.9) tells us that this is a threefold.

This example was studied mathematically in $[16,17]$. They argued that this Calabi-Yau threefold, also studied in [15], with Hodge numbers $h^{1,1}=1$ and $h^{2,1}=51$, is deformationequivalent and derived equivalent, but not birational, to an intersection of dual Grassmannians, which are equivalent to an intersection of two Pfaffians.

We shall see that this derived equivalence is reflected in the structure of the phases of our GLSM: the $r \gg 0$ phase of our GLSM will describe the self-intersection of $G(2,5)$, and the $r \ll 0$ phase will describe the self-intersection of the dual Grassmannians, realized as Pfaffian varieties. The fact that they both appear as phases of the same GLSM provides a physics argument for their derived equivalence, as the $\mathrm{B}$ model is independent of Kähler moduli.

In fact, the structure of these phases will be of further interest: in the first GLSM we study, the geometry of the $r \gg 0$ phase is realized perturbatively, but the geometry of the $r \ll 0$ phase is realized nonperturbatively via the insights of [6]. In the next subsection, we dualize both $\mathrm{SU}(2)$ gauge factors, and find the opposite: the $r \gg 0$ phase realizes the same geometry (self-intersection of $G(2,5)$ ), but does so nonperturbatively, whereas the $r \ll 0$ phase realizes the intersection of two Pfaffians perturbatively. Finally, in the subsequent subsection, we dualize on a single nonabelian gauge factor, and find that both phases realize geometry through a mix of perturbative effects (in one gauge factor) and nonperturbative effects (in the other gauge factor).

In passing, since the gauge group can also be written in the form $(\mathrm{U}(k) \times \mathrm{U}(k)) / \mathrm{U}(1)$, each with $N$ fundamentals, we could also dualize each factor to $\mathrm{U}(N-k)$, following the duality prototyped by $G(k, N) \cong G(N-k, N)$. In the present case, for $k=2, N=5$ for 
example, invoking this duality on either gauge factor separately would yield the intersection $G(2,5) \cap G(3,5)$ and phases thereof; dualizing on both factors simultaneously would yield the intersection $G(3,5) \cap G(3,5)$ and phases thereof. In either case, the resulting theories would be functionally identical to those considered here. This also shows that the choice $k=3, N=5$, consistent with the Calabi-Yau condition (3.11) does not lead to a new geometry. We thank S. Galkin for pointing this out to us.

\subsection{First GLSM}

The gauge group for this GLSM is

$$
\frac{\mathrm{U}(1) \times \mathrm{SU}(2) \times \mathrm{SU}(2)}{\mathbb{Z}_{2} \times \mathbb{Z}_{2}}
$$

where each $\mathbb{Z}_{2}$ relates the center of one $\mathrm{SU}(2)$ to a subgroup of $\mathrm{U}(1)$. The matter consists of

- $N$ chiral multiplets $\phi_{a}^{i}$ in the $(\mathbf{2}, \mathbf{1})_{\mathbf{1}}$ representation of the gauge group,

- $N$ chiral multiplets $\tilde{\phi}_{a^{\prime}}^{j}$ in the $(\mathbf{1}, \mathbf{2})_{1}$ representation of the gauge group, and

- $(1 / 2) N(N-1)$ chiral multiplets $p_{i_{1} i_{2}}=-p_{i_{2} i_{1}}$ in the $(\mathbf{1}, \mathbf{1})_{-\mathbf{2}}$ representation of the gauge group,

with superpotential

$$
W=\sum_{i_{1}<i_{2}} p_{i_{1} i_{2}}\left(f^{i_{1} i_{2}}(B)-\tilde{B}^{i_{1} i_{2}}\right)
$$

where

$$
B^{i_{1} i_{2}}=\epsilon^{a b} \phi_{a}^{i_{1}} \phi_{b}^{i_{2}}, \quad \tilde{B}^{i_{1} i_{2}}=\epsilon^{a^{\prime} b^{\prime}} \tilde{\phi}_{a^{\prime}}^{i_{1}} \tilde{\phi}_{b^{\prime}}^{i_{2}},
$$

are the 'baryons' in each of the two $\mathrm{SU}(2)$ factors of the gauge group (which mathematically define the homogeneous coordinates of the Plücker embedding $\left.G(2, N) \hookrightarrow \mathbb{P}^{(1 / 2) N(N-1)-1}\right)$, and $f^{i_{1} i_{2}}(x)=-f^{i_{2} i_{1}}(x)$ are a set of $(1 / 2) N(N-1)$ linear homogeneous polynomials in the homogeneous coordinates of $\mathbb{P}^{(1 / 2) N(N-1)-1}$. In effect, $B^{i_{1} i_{2}}$ and $\tilde{B}^{i_{1} i_{2}}$ define the Plücker embedding $G(2, N) \hookrightarrow \mathbb{P}^{(1 / 2) N(N-1)-1}$, and $f^{i_{1} i_{2}}$ describes the deformation of one of those embeddings.

Alternatively, we can write the superpotential as

$$
W=A(p)_{i_{1} i_{2}} \phi_{a}^{i_{1}} \phi_{b}^{i_{2}} \epsilon^{a b}+C(p)_{i_{1} i_{2}} \tilde{\phi}_{a^{\prime}}^{i_{1}} \tilde{\phi}_{b^{\prime}}^{i_{2}} a^{a^{\prime} b^{\prime}} .
$$

Along the lines of [6] we impose the following genericity conditions on $W$. The matrices $A_{i_{1} i_{2}} \phi_{a}^{i_{1}}$ and $C_{i_{1} i_{2}} \tilde{\phi}_{a}^{i_{1}}$ for $a=1,2$ must have maximal rank linear combinations. Furthermore we impose $A(p)_{i_{1} i_{2}} \neq C(p)_{i_{1} i_{2}}$. Under these conditions the GLSM analysis will yield the expected geometries.

For $r \gg 0$, the analysis of this GLSM is straightforward, and the result is the intersection of two copies of $G(2, N) \hookrightarrow \mathbb{P}^{(1 / 2) N(N-1)-1}$, one deformed by the linear maps $f^{i_{1} i_{2}}$, as enforced by the superpotential terms multiplied by $p_{i_{1} i_{2}}$.

Now, let us turn to the $r \ll 0$ phase. In this phase, the $\mathrm{D}$ terms prevent the $p_{i_{1} i_{2}}$ from all vanishing simultaneously, and so they form homogeneous coordinates on $\mathbb{P}^{(1 / 2) N(N-1)-1}$. 
The analysis of this phase is then closely related to that described for the Rødland model in [6]. As in the analysis there, we work in a Born-Oppenheimer approximation on the space of $p$ 's. Since $A(p)$ is an antisymmetric $N \times N$ matrix, where $N$ is odd, and antisymmetric matrices have even rank, its rank $n$ must be one of $N-1, N-3, \cdots, 0$, and since $A(p)$ acts as a mass matrix, on loci for which $A(p)$ has rank $n$, there are $N-n$ massless doublets. On loci for which it has rank $N-1$, there is one massless $\phi$ doublet in the first $\mathrm{SU}(2)$, and the vacua all run to infinity, which would leave no vacua in the IR, following [6]. If the rank of $A(p)$ is $N-3$, then there are three massless doublets of the first $\mathrm{SU}(2)$, leaving one vacuum in the IR [6]. Similarly, only loci with $C(p)$ of rank no greater than $N-3$ have vacua in the second $\mathrm{SU}(2)$.

Putting this together, we see that the $r \ll 0$ limit should be interpreted as the intersection of two Pfaffians in $\mathbb{P}^{(1 / 2) N(N-1)-1}$, where $A(p)$ has rank $\leq N-3$ and $C(p)$ has rank $\leq N-3$.

We now turn to the case $N=5$ which is of particular interest. In this case, the two Pfaffians intersecting above are both equivalent ${ }^{6}$ to Grassmannians $G(2,5)$, or more precisely, dual Grassmannians to those appearing in the $r \gg 0$ phase. In the notation of [16], the $r \gg 0$ phase describes $X=G(2,5) \cap G(2,5)$, and the $r \ll 0$ phase describes $Y$, given as the intersection of the dual Grassmannians. In [16], $X$ and $Y$ were described as 'Kanazawa double mirrors,' so-named because their mirrors were believed to be related by complex structure deformation. Here, we see that $X$ and $Y$ arise as different Kähler phases of the same GLSM - in particular, related by Kähler deformations, and so indeed their mirrors are necessarily related by complex structure deformations.

Using the parametrization of the maximal torus as discussed in section 3.2.1 the effective potential on the Coulomb branch is

$$
\begin{aligned}
\widetilde{\mathcal{W}}_{\text {eff }}= & -t\left(\alpha_{1}+\alpha_{2}\right)+\pi i\left(\alpha_{1}-\alpha_{2}\right)+\pi i\left(2 \beta_{1}-\alpha_{1}-\alpha_{2}\right) \\
& +10\left(\alpha_{1}+\alpha_{2}\right)\left(\log \left(-\alpha_{1}-\alpha_{2}\right)-1\right)-5 \alpha_{1}\left(\log \alpha_{1}-1\right)-5 \alpha_{2}\left(\log \alpha_{2}-1\right) \\
& -5 \beta_{1}\left(\log \beta_{1}-1\right)-5\left(\alpha_{1}+\alpha_{2}-\beta_{1}\right)\left(\log \left(\alpha_{1}+\alpha_{2}-\beta_{1}\right)-1\right)
\end{aligned}
$$

Defining

$$
z=\frac{\alpha_{1}}{\alpha_{2}}, \quad w_{1}=\frac{\alpha_{1}}{\beta_{1}}, \quad w_{2}=\frac{\alpha_{2}}{\beta_{1}}
$$

with $z=\frac{w_{1}}{w_{2}}$ the critical set $(3.36)$ of $\widetilde{\mathcal{W}}_{\text {eff }}$ reduces to the conditions

$$
e^{-t}=\frac{\left(z+1-\frac{1}{w_{2}}\right)^{5}}{(1+z)^{10}}, \quad z^{5}=1, \quad\left(w_{1}+w_{2}-1\right)^{5}=1 .
$$

To determine the loci of the Coulomb branches we have to remove the solutions that are fixed under the Weyl group action [6]. In this case, these are solutions satisfying $z=1$ or $w_{1}+w_{2}=2$. Removing them, we arrive at three singular points in the moduli space at

$$
e^{-t}=-1, \frac{1}{(-1+\omega)^{10}}, \frac{1}{\left(1+\omega^{2}\right)^{10}} \quad \omega=e^{\frac{2 \pi i}{10}} .
$$

This is in agreement with the data associated to the differential operator AESZ 101 in [26].

\footnotetext{
${ }^{6}$ See for example [10, section 4.2.2] for a discussion for physicists of this special case of Pfaffians and their relation to Grassmannians.
} 


\subsection{Dual description}

We now describe a dual GLSM, created by dualizing both $\mathrm{SU}(2)$ factors.

From [8, section 5.6], for an odd number $N \geq k+3$ massless fundamentals, there is a duality between the $\operatorname{Sp}(k)=\operatorname{USp}(k)$ gauge theory with $N$ fundamentals $\phi_{i}$ and $\operatorname{Sp}(N-$ $k-1$ ) gauge theory with $N$ fundamentals $\varphi_{i}$ and $(1 / 2) N(N-1)$ singlets $b_{i j}=-b_{j i}$ (dual to the baryons of the original theory), with superpotential

$$
W=\sum_{i j} b_{i j}\left[\varphi_{i} \varphi_{j}\right]=\sum_{i j} \sum_{a b} b_{i j} \varphi_{i}^{a} \varphi_{j}^{b} J_{a b}
$$

where $J$ is an antisymmetric $(N-k-1) \times(N-k-1)$ matrix of the block form

$$
J=\left[\begin{array}{cc}
0 & 1 \\
-1 & 0
\end{array}\right],
$$

as commonly arises in symplectic geometry.

Here, so long as $N \geq 5$, we can follow this prescription and dualize the first $\mathrm{SU}(2)$ with its $N$ fundamentals $\phi_{a}^{i}$ to an $\operatorname{Sp}(N-3)$ gauge theory with $N$ fundamentals $\varphi_{i}^{a}$ and $(1 / 2) N(N-1)$ singlets $b^{i j}=-b^{j i}$. The second $\mathrm{SU}(2)$ dualizes to an $\operatorname{Sp}(N-3)$ gauge theory with $N$ fundamentals $\tilde{\varphi}_{i}^{a^{\prime}}$ and singlets $\tilde{b}^{i j}$. The $\mathrm{U}(1)$ factor is unchanged, and as the center of $\operatorname{Sp}(n)$ is ${ }^{7} \mathbb{Z}_{2}$, the finite group quotient is also unchanged, hence the gauge group of the dual theory is

$$
\frac{\mathrm{U}(1) \times \operatorname{Sp}(N-3) \times \operatorname{Sp}(N-3)}{\mathbb{Z}_{2} \times \mathbb{Z}_{2}} .
$$

The dual superpotential has the form

$$
\begin{aligned}
W & =\left(A(p)_{i_{1} i_{2}}+\varphi_{i_{1}}^{a} \varphi_{i_{2}}^{b} J_{a b}\right) b^{i_{1} i_{2}}+\left(C(p)_{i_{1} i_{2}}+\tilde{\varphi}_{i_{1}}^{a^{\prime}} \tilde{\varphi}_{i_{2}}^{b^{\prime}} J_{a^{\prime} b^{\prime}}\right) \tilde{b}^{i_{1} i_{2}}, \\
& =\sum_{i_{1}<i_{2}} p_{i_{1} i_{2}}\left(f^{i_{1} i_{2}}(b)-\tilde{b}^{i_{1} i_{2}}\right)+\varphi_{i_{1}}^{a} \varphi_{i_{2}}^{b} J_{a b} b^{i_{1} i_{2}}+\tilde{\varphi}_{i_{1}}^{a_{1}^{\prime}} \tilde{\varphi}_{i_{2}}^{b^{\prime}} J_{a^{\prime} b^{\prime}} \tilde{b}^{i_{1} i_{2}} .
\end{aligned}
$$

Since the $b^{i j}$ and $\tilde{b}^{i j}$ are dual to baryons, they have gauge $\mathrm{U}(1)$ charge 2 . The form of superpotential couplings implies that $\varphi_{i}^{a}, \tilde{\varphi}_{i}^{a^{\prime}}$ have $\mathrm{U}(1)$ charge -1 . Finally, the $p_{i j}$ have the same $\mathrm{U}(1)$ charge as in the original theory, namely -2 .

From counting $\mathrm{U}(1)$ charges, it is straightforward to check that this intersection will be Calabi-Yau only in the special case that $N=5$, as expected.

Calling the FI-theta parameter $\tilde{t}=\tilde{r}-i \tilde{\theta}$, the phase $\tilde{r} \gg 0$, D terms imply that the $b^{i j}$ and $\tilde{b}^{i j}$ are not all zero. The pertinent form of the superpotential is

$$
W=\sum_{i_{1}<i_{2}} p_{i_{1} i_{2}}\left(f^{i_{1} i_{2}}(b)-\tilde{b}^{i_{1} i_{2}}\right)+\varphi_{i_{1}}^{a} \varphi_{i_{2}}^{b} J_{a b} b^{i_{1} i_{2}}+\tilde{\varphi}_{i_{1}}^{a_{1}^{\prime}} \tilde{\varphi}_{i_{2}}^{b^{\prime}} J_{a^{\prime} b^{\prime}} \tilde{b}^{i_{1} i_{2}}
$$

In the previous GLSM, the $r \gg 0$ phase could be understood perturbatively, but in this model, we must analyze this phase nonperturbatively, following [6], combined with the

\footnotetext{
${ }^{7}$ See for example [27, appendix A] for a more exhaustive discussion of centers of Lie groups.
} 
same assumptions about $f$ as in the original model. We work in a Born-Oppenheimer approximation across the space of $b$ 's, $\tilde{b}$ 's, and interpret the terms

$$
\varphi_{i_{1}}^{a} \varphi_{i_{2}}^{b} J_{a b} b^{i_{1} i_{2}}+\tilde{\varphi}_{i_{1}}^{a_{1}^{\prime}} \tilde{\varphi}_{i_{2}}^{b^{\prime}} J_{a^{\prime} b^{\prime}} \tilde{b}^{i_{1} i_{2}}
$$

as $b$ - and $\tilde{b}$-dependent mass matrices for $\varphi$ and $\tilde{\varphi}$.

For the case $N=5$, the gauge group has two $\mathrm{SU}(2)$ factors, and so we can apply the results of [6] to argue that the only contribution to the vacua will arise from the locus where the (antisymmetric) $N \times N$ mass matrices have rank 2, so that there are three massless doublets and hence one vacuum in the IR. (On the rank 4 locus, there is one massless doublet, and no vacua in the IR [6].) Thus, from each $\mathrm{SU}(2)$ factor, we get a Pfaffian variety, over the (cone over) a copy of $\mathbb{P}^{9}$ determined by $\left(b^{i j}\right)$ or $\left(\tilde{b}^{i j}\right)$. (To be clear, at this point in the analysis, the $b$ 's and $\tilde{b}$ 's do not separately describe $\mathbb{P}^{9}$ 's, only the product of cones over copies of $\mathbb{P}^{9}$. In the next step, when we take the intersection, the result will be equivalent to working in a single copy of $\mathbb{P}^{9}$.) Furthermore, each of these two Pfaffians is equivalent to $G(2,5)$ [10, section 4.2.2], and the remaining superpotential term

$$
p_{i_{1} i_{2}}\left(f^{i_{1} i_{2}}(b)-\tilde{b}^{i_{1} i_{2}}\right)
$$

dictates that we should take the intersection of one copy of $G(2,5)$ with a linear rotation of the other copy of $G(2,5)$, following the same analysis as described previously in this paper.

As a result, for $N=5$, for $\tilde{r} \gg 0$, we recover the same geometry — a self-intersection of $G(2,5)$ with linear rotation - that we recovered in the previous GLSM, albeit in this GLSM, the geometry appears wholly by nonperturbative effects as in [6], rather than perturbatively.

Next we consider the phase $\tilde{r} \ll 0$. In this phase, D-terms imply that not all of the $\varphi_{i}^{a}$. $\tilde{\varphi}_{i}^{a^{\prime}}$, and $p_{i_{1} i_{2}}$ 's can vanish. The pertinent form for the superpotential is

$$
W=\left(A(p)_{i_{1} i_{2}}+\varphi_{i_{1}}^{a} \varphi_{i_{2}}^{b} J_{a b}\right) b^{i_{1} i_{2}}+\left(C(p)_{i_{1} i_{2}}+\tilde{\varphi}_{i_{1}}^{a^{\prime}} \tilde{\varphi}_{i_{2}}^{b^{\prime}} J_{a^{\prime} b^{\prime}}\right) \tilde{b}^{i_{1} i_{2}} .
$$

This is precisely an intersection of two PAXY models, each describing a Pfaffian for the locus of rank $N-3$ of a matrix $(A(p), C(p))$ in the projective space defined by the $p$ 's, as described in [8] and [9, section 3.5]. These were described in those references as $\operatorname{Sp}(N-3)$ gauge theories with a superpotential of the form

$$
b^{i j}\left(A_{i j}-x_{i}^{a} x_{j}^{b} J_{a b}\right)
$$

where the $b^{i j}$ are chiral superfields multiplying pertinent superpotential terms, and the $x_{i}^{a}$ are introduced to enforce the rank constraint on the antisymmetric matrix $A$. The theory here in the $\tilde{r} \ll 0$ phase is explicitly an intersection of two such PAXY models for Pfaffians, as analyzed previously in section 3.3 .

In the case $N=5$, from [10, section 4.2.2], each Pfaffian can be interpreted as a copy of $G(2,5)$, or more precisely a dual, so we immediately recover the geometric interpretation of the $r \ll 0$ phase of the previous GLSM for this geometry, as expected. In the previous 
GLSM, the $r \ll 0$ phase was understood nonperturbatively using the methods of [6]; here, by contrast, the phase (in the dual GLSM) can be understood entirely perturbatively.

Another non-trivial check of the duality is to compare the Coulomb branch analysis of this model with the original one in the Calabi-Yau case. Upon identification of the FI-theta parameters of the original theory and its dual, one should obtain the same result for the singular points in the Kähler moduli space. Since the dual gauge group is the same as the original one, we can use the same parametrization of the maximal torus, as discussed in section 3.2.1. Denoting the FI-theta parameter of the dual theory by $\tilde{t}$ one finds

$$
\begin{aligned}
\widetilde{\mathcal{W}}_{\text {eff }}= & -\tilde{t}\left(\alpha_{1}+\alpha_{2}\right)+\pi i\left(\alpha_{1}-\alpha_{2}\right)+\pi i\left(2 \beta_{1}-\alpha_{1}-\alpha_{2}\right) \\
& +10\left(\alpha_{1}+\alpha_{2}\right)\left(\log \left(-\alpha_{1}-\alpha_{2}\right)-1\right)+5 \alpha_{1}\left(\log \left(-\alpha_{1}\right)-1\right)+5 \alpha_{2}\left(\log \left(-\alpha_{2}\right)-1\right) \\
& +5 \beta_{1}\left(\log \left(-\beta_{1}\right)-1\right)+5\left(\alpha_{1}+\alpha_{2}-\beta_{1}\right)\left(\log \left(-\alpha_{1}-\alpha_{2}+\beta_{1}\right)-1\right) \\
& -20\left(\alpha_{1}+\alpha_{2}\right)\left(\log \left(\alpha_{1}+\alpha_{2}\right)-1\right)
\end{aligned}
$$

Note that the last line accounts for the contribution of the additional singlet fields $b^{i_{1} i_{2}}$ and $\tilde{b}^{i_{1} i_{2}}$, where we have once again used $\alpha_{1}+\alpha_{2}=\beta_{1}+\beta_{2}$. With (4.6), the critical locus is determined by

$$
e^{-\tilde{t}}=\frac{(1+z)^{10}}{\left(1+z-\frac{1}{w_{2}}\right)^{5}}, \quad z^{5}=1, \quad\left(w_{1}+w_{2}-1\right)^{5}=1
$$

Comparing with (4.7), these are exactly the same conditions if we identify $e^{t}=e^{-\tilde{t}}$. Hence, the singular points in the Kähler moduli space of the two models are identical, giving evidence that we indeed have two equivalent GLSM realizations of the same pair of CalabiYaus.

\subsection{Dualization on a single factor}

In the previous subsection, we analyzed the result of dualizing on both $\mathrm{SU}(2)$ factors. For completeness, here we briefly outline the analysis of a duality on a single $\mathrm{SU}(2)$ factor.

Following the previous analysis, the dual GLSM has gauge group

$$
\frac{\mathrm{U}(1) \times \mathrm{Sp}(N-3) \times \mathrm{SU}(2)}{\mathbb{Z}_{2} \times \mathbb{Z}_{2}}
$$

with

- $N$ chiral multiplets $\varphi_{i}^{a}$ in the $(\mathbf{N}-\mathbf{3}, \mathbf{1})_{-1}$ representation,

- $(1 / 2) N(N-1)$ chiral multiplets $b^{i j}=-b^{j i}$ in the $(\mathbf{1}, \mathbf{1})_{2}$ representation,

- $N$ chiral multiplets $\tilde{\phi}_{a^{\prime}}^{j}$ in the $(\mathbf{1}, \mathbf{2})_{1}$ representation,

- $(1 / 2) N(N-1)$ chiral multiplets $p_{i j}=-p_{j i}$ in the $(\mathbf{1}, \mathbf{1})_{-2}$ representation, 
with superpotential

$$
\begin{aligned}
W & =\sum_{i_{1}<i_{2}}\left(A(p)_{i_{1} i_{2}}+\varphi_{i_{1}}^{a} \varphi_{i_{2}}^{b} J_{a b}\right) b^{i_{1} i_{2}}+\sum_{i_{1}<i_{2}} C(p)_{i_{1} i_{2}} \tilde{\phi}_{a^{\prime}}^{i_{1}} \tilde{\phi}_{b^{\prime}}^{i_{2}} \epsilon^{a^{\prime} b^{\prime}} \\
& =\sum_{i_{1}<i_{2}} p_{i_{1} i_{2}}\left(f^{i_{1} i_{2}}(b)-\tilde{B}^{i_{1} i_{2}}\right)+\varphi_{i_{1}}^{a} \varphi_{i_{2}}^{b} J_{a b} b^{i_{1} i_{2}}
\end{aligned}
$$

where $\tilde{B}$ is defined as previously.

Let us look in more detail at the gauge group and the quotient. First write $\alpha \in \mathrm{U}(1)$, $g_{1} \in \mathrm{USp}(N-3), g_{2} \in \mathrm{SU}(2)$. Then:

$$
p_{i j} \rightarrow \alpha^{-2} p_{i j} \quad b^{i j} \rightarrow \alpha^{2} b^{i j} \quad \varphi_{i} \rightarrow \alpha^{-1} g_{1} \varphi_{i} \quad \tilde{\phi}^{j} \rightarrow \alpha g_{2} \phi^{j} .
$$

Assigning the FI parameter $\hat{r}$ to $\mathrm{U}(1)$ the $\mathrm{U}(1) \mathrm{D}$-term is

$$
2 \sum_{k}\left(-\left|p_{k}\right|^{2}+\left|b_{k}\right|^{2}\right)+\sum_{i}\left(-\left|\varphi_{i}^{a}\right|^{2}+\left|\tilde{\phi}_{b}^{j}\right|^{2}\right)=2 \hat{r}
$$

This means that at $\hat{r} \gg 0$ not all $\tilde{\phi}$ and $b$ are allowed to vanish whereas at $\hat{r} \ll 0$ not all $p$ and $\varphi$ are allowed to vanish simultaneously. This is weaker than in the original model: here the D-terms allow for branches where $b=0$ or $\tilde{\phi}=0$ at $\hat{r} \gg 0$ and $p=0$ or $\varphi=0$ at $\hat{r} \ll 0$. This looks like there can be a mixture of strongly coupled and weakly coupled components in either phase. Such phenomena have also been observed in [12].

Let us first consider the phase $\hat{r} \gg 0$. In this phase, the U(1) D-terms imply that not all the $b^{i j}$ or $\tilde{\phi}_{a^{\prime}}^{j}$ can vanish. In this phase, it is most useful to write the superpotential in the form

$$
W=\sum_{i_{1}<i_{2}} p_{i_{1} i_{2}}\left(f^{i_{1} i_{2}}(b)-\tilde{B}^{i_{1} i_{2}}\right)+\varphi_{i_{1}}^{a} \varphi_{i_{2}}^{b} J_{a b} b^{i_{1} i_{2}}
$$

The first terms, $p(f(b)-\tilde{B})$, appear to give a perturbative realization of the intersection of the Plücker embedding of $G(2, N)$ (via the $\tilde{\phi}$ ) with a rotation of the projective space $\mathbb{P}^{(1 / 2) N(N-1)-1}$ (defined by the $b$ 's).

The second terms, $[\varphi, \varphi]_{i j} b^{i j}$, can be interpreted nonperturbatively. Working in a BornOppenheimer approximation on $\mathbb{P}^{(1 / 2) N(N-1)-1}$, this is a mass term for the $\varphi$ 's. Following the same logic as [6] and previously, there are no vacua where the matrix $[\varphi, \varphi]$ has rank $N-1$, only where it has rank $N-3$ or less. In this fashion, we find that the second terms dictate an intersection with a Pfaffian variety in $\mathbb{P}^{(1 / 2) N(N-1)-1}$.

For $N=5$, this Pfaffian is the same as the Grassmannian $G(2,5)$ [10, section 4.2.2]. As a result, this phase describes the self-intersection of $G(2,5)$ with a linear rotation, matching the result for the duality frames, albeit here with a mix of perturbative and nonperturbative analysis.

Next, let us consider the other phase, $\hat{r} \ll 0$. In this phase, not all the $\varphi_{i}^{a}$ or $p_{i j}$ can vanish. The superpotential is most usefully written in the form

$$
W=\sum_{i_{1}<i_{2}}\left(A(p)_{i_{1} i_{2}}+\varphi_{i_{1}}^{a} \varphi_{i_{2}}^{b} J_{a b}\right) b^{i_{1} i_{2}}+\sum_{i_{1}<i_{2}} C(p)_{i_{1} i_{2}} \tilde{\phi}_{a^{\prime}}^{i_{1}} \tilde{\phi}_{b^{\prime}}^{i_{2}} a^{a^{\prime} b^{\prime}}
$$


In this phase, the first set of terms, $(A(p)+[\varphi, \varphi]) b$, appear to give a perturbative realization of an intersection. For $N=5$, this is the intersection of a Grassmannian $G(2,5)$ (whose baryons are $[\varphi \varphi]$ ) and a rotation of the projective space $\mathbb{P}^{(1 / 2) N(N-1)-1}$ (defined by the $p$ 's).

The second set of terms, $C(p) \tilde{B}$, can be interpreted nonperturbatively. Working in a Born-Oppenheimer approximation on $\mathbb{P}^{(1 / 2) N(N-1)-1}$, this is a mass term for the $\tilde{\phi}$ 's. Following the same logic as [6] and previously, there are no vacua where the matrix $\tilde{B}^{i j}$ has rank $N-1$, only where it has rank $N-3$ or less. In this fashion, we find that the second terms dictate an intersection with a Pfaffian variety in $\mathbb{P}^{(1 / 2) N(N-1)-1}$.

For $N=5$, for the same reasons as above, the Pfaffian coincides with the (dual) Grassmannian, and so again we recover the same geometry in the phase $\hat{r} \ll 0$ as in other phases, albeit again via two contributions, one perturbative, the other nonperturbative.

It is interesting to see how applying the duality only to one of the non-abelian factors of the gauge group affects the Coulomb branch analysis in the Calabi-Yau case. Introducing an FI-theta parameter $\hat{t}$ we get

$$
\begin{aligned}
\widetilde{\mathcal{W}}_{\mathrm{eff}}= & -\hat{t}\left(\alpha_{1}+\alpha_{2}\right)+\pi i\left(\alpha_{1}-\alpha_{2}\right)+\pi i\left(2 \beta_{1}-\alpha_{1}-\alpha_{2}\right) \\
& +10\left(\alpha_{1}+\alpha_{2}\right)\left(\log \left(-\alpha_{1}-\alpha_{2}\right)-1\right)+5 \alpha_{1}\left(\log \left(-\alpha_{1}\right)-1\right)+5 \alpha_{2}\left(\log \left(-\alpha_{2}\right)-1\right) \\
& -5 \beta_{1}\left(\log \left(\beta_{1}\right)-1\right)-5\left(\alpha_{1}+\alpha_{2}-\beta_{1}\right)\left(\log \left(\alpha_{1}+\alpha_{2}-\beta_{1}\right)-1\right) \\
& -10\left(\alpha_{1}+\alpha_{2}\right)\left(\log \left(\alpha_{1}+\alpha_{2}\right)-1\right) .
\end{aligned}
$$

Using the definitions (4.6) the critical locus is determined by

$$
e^{-\hat{t}}=-\left(1+z-\frac{1}{w_{2}}\right)^{5}, \quad z^{5}=1, \quad\left(w_{1}+w_{2}-1\right)^{5}=1
$$

The second and third equations are the same as in the other two models, the first one has a slightly simpler form, but one still finds the same solutions for the location of the Coulomb branch as in the original model under the identification $e^{-\hat{t}}=e^{-t}$.

\section{Other Kanazawa Calabi-Yaus}

The intersection of two copies of $G(2,5)$, discussed in the previous section, is one of several examples of Calabi-Yau threefolds constructed in [15], listed there as $X_{25}$. Some of the examples discussed there are already in the physics literature: the example labeled $X_{14}$ is Rødland's model [6,28], and $X_{5,7,10,13}$ were discussed in [11]. In this section, we will discuss physical realizations of the remaining examples.

\section{$5.1 \quad Y_{5}, Y_{10}$}

In [15] Kanazawa gives two examples (examples 2.17 and 2.18, label-led there $Y_{10}$ and $Y_{5}$, respectively) of Calabi-Yaus that are complete intersections of Pfaffians with a hypersurface of degree $d$ in a weighted projective space of dimension $M-1$ and weights $q_{k}$. We propose that both examples can be realized in terms of a GLSM with gauge group $(\mathrm{U}(1) \times \mathrm{USp}(2)) / \mathbb{Z}_{2} \simeq \mathrm{U}(2)$. The matter content is 
- $M$ chiral superfields $\phi_{k}$ in representation $\mathbf{1}_{2 q_{k}}$, the first $m$ having $q_{k}=1$

- 5 chiral superfields $x_{i}^{a}$ in representation $\mathbf{2}_{1}$,

- 10 chiral superfields $b^{i j}=-b^{j i}$ in representation $\mathbf{1}_{-2}$,

- 1 chiral superfield $q$ in representation $\mathbf{1}_{-2 d}$.

The superpotential is

$$
W=b^{i j}\left(A(\phi)_{i j}-x_{i}^{a} x_{j}^{b} \epsilon_{a b}\right)+q f_{d}(\phi),
$$

where $A(\phi)$ is a skew-symmetric $5 \times 5$ matrix (whose rank two locus defines the Pfaffian), with entries of charge 2 under the $\mathrm{U}(1)$, and $f_{d}(\phi)$ is a polynomial of degree $d$ (hence charge $2 d)$. In the following we will assume that the Calabi-Yau condition is satisfied. For later reference, we also state the F-term equations explicitly:

$$
\begin{aligned}
& 0=A_{i j}(\phi)-x_{i}^{a} x_{j}^{b} \epsilon_{a b} \\
& 0=b^{i j} \frac{\partial A_{i j}(\phi)}{\partial \phi_{k}}+q \frac{\partial f_{d}(\phi)}{\partial \phi_{k}} \\
& 0=b^{i j} x_{i}^{a} \\
& 0=f_{d}(\phi)
\end{aligned}
$$

There is a large volume phase $r \gg 0$ where the D-terms imply that $\phi_{1, \ldots, M}$ and $x_{1, \ldots, 5}$ are not allowed to vanish simultaneously and the matrix $x_{i}^{a}$ has rank 2 . The vacuum is a complete intersection of a Pfaffian with a degree $d$ hypersurface given by

$$
A_{i j}\left(\phi_{k}\right)-x_{i}^{a} x_{j}^{b} \epsilon_{a b}=0 \quad f_{d}\left(\phi_{k}\right)=0 \quad q, b^{i j}=0 .
$$

In the $r \ll 0$-phase $q$ and $b^{i j}$ are not allowed to vanish simultaneously. There is a vacuum where $\phi_{k}, x$ and $b^{i j}$ are zero and $q$ obtains a vev. The U(1)-symmetry is broken to $\mathbb{Z}_{d}$. Small fluctuations of the $\phi_{k}$ generate a potential, indicating the structure LandauGinzburg orbifold. However, this cannot not the whole story since also fluctuations of $x$ and $b^{i j}$ have to be taken into account, and we expect that this phase is an exotic type of hybrid model. We will not be able to present a full discussion of this exotic phase, but we can extract some further properties if consider the Hori dual. The dual model has the same non-abelian factor. There are no $b^{i j}$, and the $x_{i}$ have the opposite $\mathrm{U}(1)$-charges compared to the original theory, while the $\phi_{k}$ and $q$ remain unchanged. The superpotential is

$$
W=A^{i j}\left(\phi_{k}\right) x_{i}^{a} x_{j}^{b} \epsilon_{a b}+q f_{d}\left(\phi_{k}\right) .
$$

The F-term equations are

$$
\begin{aligned}
& 0=\frac{\partial A_{i j}(\phi)}{\partial \phi_{k}} x_{i}^{a} x_{j}^{b} \epsilon_{a b}+q \frac{\partial f_{d}(\phi)}{\partial \phi_{k}} \\
& 0=A^{i j}\left(\phi_{k}\right) x_{i}^{a} \\
& 0=f_{d}(\phi) .
\end{aligned}
$$


For $\tilde{r} \gg 0$ we recover the complete intersection of $f_{d}(\phi)$ where the Pfaffian is now realized non-perturbatively. Now consider the $\tilde{r} \ll 0$ phase where $q$ and $x$ are not allowed to vanish simultaneously. For $q=0$ and $\phi_{k}=0$ there is a solution to the D-term and F-term equations governed by

$$
A_{l}^{i j} x_{i}^{a} x_{j}^{b} \epsilon_{a b}=0, \quad l=1, \ldots, m,
$$

where $l$ labels the $m \phi_{k}$ with $q_{k}=1$. This is a complete intersection of codimension $m$ in $G(2,5)$. This is only Calabi-Yau for $m=5$, which is not the case for the examples of interest. Therefore, this branch of solutions to the D- and F-term equations, if it exists, is expected to have properties of hybrid-type configuration upon turning on classical fluctuations of the $\phi_{k}$ and $q$. Further note that for $m>6$ a count of dimensions shows that $x=0$. As we have seen in the original model, there is also a second branch where $q \neq 0$, the $\mathrm{U}(1)$ broken to $\mathbb{Z}_{d}$. Phases where the D-term and F-terms equations admit more than one branch of solutions have already been observed in $[11,12]$ and have been linked to "bad" hybrid models in the sense of [29].

Aside from the solutions to the D-term and F-term equations presented here, there may be further ones, depending on the concrete choice of model. Indeed, we will find a non-compact branch for the model $Y_{5}$ below, indicating a singularity.

\subsection{1 $\quad Y_{10}$}

This example is a Calabi-Yau threefold, constructed mathematically as the intersection of a Pfaffian associated to $\mathcal{E} \equiv \mathcal{O}^{5} \rightarrow \mathbb{P}_{\left[1^{7}, 2\right]}^{7}$, (in the sense that the matrix defining the Pfaffian is a section of $\wedge^{2} \mathcal{E}(t)$ for some $t$,) with a quartic hypersurface in $\mathbb{P}_{\left[1^{7}, 2\right]}^{7}$. It is discussed in [15, example 2.17].

We propose that the corresponding GLSM is given as follows. Starting off with a gauge group $\mathrm{U}(2)=\frac{\mathrm{U}(1) \times \mathrm{SU}(2)}{\mathbb{Z}_{2}}$, the matter content is

- 7 chiral superfields $\phi_{i}$ in representation $\mathbf{1}_{2}$,

- 1 chiral superfield $\phi_{8}$ in representation $\mathbf{1}_{4}$, forming the remaining homogeneous coordinate on $\mathbb{P}_{\left[1^{7}, 2\right]}^{7}$,

- 5 chiral superfields $x_{i}^{a}$ in representation $\mathbf{2}_{1}$,

- 10 chiral superfields $b^{i j}=-b^{j i}$ in representation $\mathbf{1}_{-2}$,

- 1 chiral superfield $q$ in representation $\mathbf{1}_{-8}$,

and superpotential

$$
W=b^{i j}\left(A(\phi)_{i j}-x_{i}^{a} x_{j}^{b} \epsilon_{a b}\right)+q f_{4}(\phi)
$$

where $A(\phi)$ is a skew-symmetric $5 \times 5$ matrix (whose rank two locus defines the Pfaffian), with entries of charge 2 under the $\mathrm{U}(1)$, and $f_{4}(\phi)$ is a generic polynomial of degree four (hence charge 8).

The Calabi-Yau condition is satisfied: $-2 \cdot 7-4 \cdot 1+8 \cdot 1-3 \cdot 2 \cdot 5+10 \cdot 4=0$. Now we implement the $\mathbb{Z}_{2}$ quotient to rewrite the gauge group as $\mathrm{U}(2)$. With $\alpha \in \mathrm{U}(1)$, 
$g \in \mathrm{SU}(2)$ and $\tilde{g}=\alpha g \in \mathrm{U}(2)$ the fields $\phi_{1, \ldots, 7}$ transform in the det-representation and $\phi_{8}$ in the $\operatorname{det}^{2}$-representation. The $x$-fields transform in the fundamental and $q$ and $b^{i j}$ are in the $\operatorname{det}^{-4}$ and $\operatorname{det}^{-2}$ representations, respectively.

The entries of the matrix $A(p)$ are of degree 2 in the $\phi_{k}$, i.e. linear in $\phi_{1, \ldots, 7}$ while $\phi_{8}$ does not appear at all. In the $r \gg 0$ phase we find a geometry (5.6). We do not find any non-compact directions. To see this, note in particular that the rank of $A(\phi)$ drops to zero if $\phi_{1, \ldots, 7}=0$, indicating a singularity. However, then the F-term (5.5) implies $\phi_{8}=0$ and the remaining $\mathrm{F}$-terms enforce $x=0$ which is disallowed by the D-terms in the phase $r \gg 0$. Reference [15] also points out that this model coincides mathematically with a double covering of a Fano threefold. We have not seen this description directly in physics, but it would be interesting to do so.

In the $r \ll 0$-phase, respectively the $\tilde{r} \ll 0$ phase of the dual, there is certainly a solution where $q \neq 0$ and all other fields zero. The branch $q=0, \phi_{k}=0$ does not seem to exist for dimensional reasons.

Computing the effective potential on the Coulomb branch, one finds two singular points in the Kähler moduli space.

$$
e^{-t}=-\frac{1}{64} \frac{1}{(1-\omega)^{5}},-\frac{1}{64} \frac{1}{\left(1+\omega^{2}\right)^{5}} \quad \omega=e^{\frac{2 \pi i}{10}} .
$$

This, together with the topological data given in [15] can be found under the label AESZ 51 in the Calabi-Yau operator database [26]. The information in the database also gives further evidence that the $r \ll 0$ phase is a "bad" hybrid: the Riemann symbol indicates the same structure of the periods as observed in [29].

\section{$5.1 .2 \quad Y_{5}$}

This example is a Calabi-Yau threefold, constructed mathematically as the intersection of a Pfaffian associated to $\mathcal{O}^{5} \rightarrow \mathbb{P}_{\left[1^{6}, 2,3\right]}^{7}$ with a degree 6 hypersurface in $\mathbb{P}_{\left[1^{6}, 2,3\right]}^{7}$. It is described in [15, example 2.18].

We propose that this model is described by the following GLSM, with gauge group $\mathrm{U}(2)=\frac{\mathrm{U}(1) \times \mathrm{SU}(2)}{\mathbb{Z}_{2}}$, and matter

- 6 chiral superfields $\phi_{i}$ in representation $\mathbf{1}_{2}$,

- 1 chiral superfield $\phi_{7}$ in representation $\mathbf{1}_{4}$,

- 1 chiral superfield $\phi_{8}$ in representation $\mathbf{1}_{6}$,

- 5 chiral superfields $x_{i}^{a}$ in representation $\mathbf{2}_{1}$,

- 10 chiral superfields $b^{i j}=-b^{j i}$ in representation $\mathbf{1}_{-2}$,

- 1 chiral superfield $q$ in representation $\mathbf{1}_{-12}$.

and superpotential

$$
W=b^{i j}\left(A(\phi)_{i j}-x_{i}^{a} x_{j}^{b} \epsilon_{a b}\right)+q f_{6}(\phi),
$$


where $A(\phi)$ is a skew-symmetric $5 \times 5$ matrix (whose rank two locus defines the Pfaffian), with entries of charge 2 under the $\mathrm{U}(1)$, and $f_{6}(\phi)$ is a polynomial of degree six (hence charge 12). The sum of the U(1) charges vanishes, consistent with the Calabi-Yau condition, and the superpotential above is explicitly gauge-invariant. Note that $\phi_{7,8}$ do not appear in $A(\phi)$. The analysis of the phases is indicated as above, except that we find a non-compact direction. To see this, consider the locus $\phi_{1, \ldots, 6}=0$ in the $r \gg 0$ phase. Then it follows from the F-terms (5.2), (5.4), and the first six of (5.3) that $b^{i j}=0$ and $x=0$. The remaining F-terms (5.5) and the last two of (5.3) then have a solution, namely $q=0$, where $\phi_{8}^{2} \sim \phi_{7}^{3} \neq 0$. This result is consistent with [15] where it was conjectured that no smooth example of this form exists.

Again, we cannot say much about the other phase of this model. The analysis of the dual theory shows that two branches, one with $q=0$, one with $q \neq 0$ exist. This indicates that this phase is a "bad" hybrid model.

The Coulomb branch analysis yields two singular points at

$$
e^{-t}=-\frac{1}{432} \frac{1}{(1-\omega)^{5}},-\frac{1}{64} \frac{1}{\left(1+\omega^{2}\right)^{5}} \quad \omega=e^{\frac{2 \pi i}{10}}
$$

This, together with the topological data given in [15] can be found under the label AESZ 63 in the Calabi-Yau operator database [26]. As for $Y_{10}$, the Riemann symbol supports the claim that the non-geometric phase is an exotic hybrid.

\section{$5.2 \quad X_{9}$}

This is a Pfaffian associated to $\mathcal{E} \equiv \mathcal{O}(2) \oplus \mathcal{O}(1)^{2} \oplus \mathcal{O}^{2} \rightarrow \mathbb{P}_{\left[1^{6}, 2\right]}^{6}$. It's isomorphic to $\mathbb{P}^{5}[3,3]$.

We propose that the corresponding GLSM has gauge group $\mathrm{U}(1) \times \mathrm{SU}(2)$, with matter

- 6 chiral superfields $\phi_{i}$ in representation $\mathbf{1}_{1}$,

- 1 chiral superfield $\phi_{7}$ in representation $\mathbf{1}_{2}$,

- 5 chiral superfields $x_{i}^{a}$ in representations $\mathbf{2}_{2}, \mathbf{2}_{1}, \mathbf{2}_{1}, \mathbf{2}_{0}, \mathbf{2}_{0}$,

- 10 chiral superfields $b^{i j}=-b^{j i}$ that are $\mathrm{SU}(2)$ singlets of charges

$$
-3,-3,-2,-2,-2,-1,-1,-1,-1,0 \text {, }
$$

and superpotential

$$
W=b^{i j}\left(A(\phi)_{i j}-x_{i}^{a} x_{j}^{b} \epsilon_{a b}\right)
$$

where $A(\phi)$ is a $5 \times 5$ skew-symmetric matrix which should be interpreted as a section of $\wedge^{2} \mathcal{E}$. (An example of such a matrix $A$, in fact a one-parameter family of such matrices, is given in [15].) The sum of the $\mathrm{U}(1)$ charges vanishes, consist with the Calabi-Yau condition, and the superpotential above is explicitly gauge-invariant. 


\section{Conclusions}

In this paper we have described GLSMs for several further exotic constructions. We began by describing GLSM realizations of Veronese, Segre, and diagonal embeddings, which can be useful building-blocks for various constructions of Calabi-Yau. We then described GLSMs for intersections of Grassmannians, after linear rotations and Veronese and Segre embeddings. We then discussed the (non-birational) phases of the intersections of two Grassmannians with a linear rotation, giving a physical realization of mathematical structures recently discussed in $[16,17]$. We then concluded with GLSMs for some other exotic Calabi-Yau constructions in [15].

We observed at one point that the GLSM for the Veronese map coincides with the PAXY model of [9] describing the image as a (hyper)determinantal variety. Now, PAXY and PAX models exist in greater generality, in which fields $x, y$ are introduced to realize a determinantal variety. To our mind this begs the question of whether those fields can be understood in terms of defining maps in a fashion analogous to what we have seen here for the Veronese embeddings. We leave this matter for future work.

Analogous mathematical phenomena are described in [30] for spinor varieties, and in [31] for cubic fourfolds. Further, more exotic mathematical examples can also be found in $[32$, section 6$]$, $[33,34]$. It would be very interesting to construct corresponding GLSMs.

A further interesting class of examples to look at are Calabi-Yaus that do not have a point of maximal unipotent monodromy as discussed for instance in [35-37]. This would translate into GLSMs that do not have geometric phases. In such cases localization techniques from the GLSM that do not rely on mirror constructions may be particularly useful for analyzing these Calabi-Yaus.

Most known examples of non-abelian GLSMs have matter that transforms either in the fundamental or some one-dimensional representation. In section 2.4 we have discussed GLSMs with matter in the $\operatorname{Sym}^{\mathbf{d}} \mathbf{k}$ representation. It would be interesting to analyze GLSMs with exotic matter in further detail, as they will lead to interesting new examples of Calabi-Yau spaces.

Finally, the construction of intersecting Grassmannians seems to fit into a more general framework [38]. It would be interesting to see if this approach leads to a systematic construction of a larger class of new Calabi-Yaus via non-abelian GLSMs.

While the main goal of this work was to identify GLSMs that lead to certain classes of Fanos and Calabi-Yaus, it is possible to extract further (quantum) information from GLSMs. In the Calabi-Yau case methods from supersymmetric localization can be applied to compute Gromov-Witten invartiants [1], the elliptic genus [2], the central charge of B-type D-branes [3,4] or Yukawa couplings [5]. While these methods can also work in non-Calabi-Yau GLSMs, the interpretation of the results is less clear. In the Fano case, one can still use the GLSM to compute certain quantities that are invartiant under RG flow, by taking the A-twist of the theory (if the $\mathrm{U}(1)_{V}$ symmetry is non-anomalous). An example of such an invariant that can be computed via the A-twisted GLSM is the quantum cohomology ring of a Fano non-linear sigma model. We plan to return to this in future work. 


\section{Acknowledgments}

We would like to thank S. Galkin, S. Katz, I. Melnikov, D. Morrison, T. Pantev, J. Rennemo, M. Romo, S. Sam, E. Scheidegger and E. Segal for useful conversations. E.S. was partially supported by NSF grants PHY-1417410 and PHY-1720321.

Open Access. This article is distributed under the terms of the Creative Commons Attribution License (CC-BY 4.0), which permits any use, distribution and reproduction in any medium, provided the original author(s) and source are credited.

\section{References}

[1] H. Jockers, V. Kumar, J.M. Lapan, D.R. Morrison and M. Romo, Two-sphere partition functions and Gromov-Witten invariants, Commun. Math. Phys. 325 (2014) 1139 [arXiv: 1208.6244] [INSPIRE].

[2] F. Benini, R. Eager, K. Hori and Y. Tachikawa, Elliptic genera of $2 d \mathcal{N}=2$ gauge theories, Commun. Math. Phys. 333 (2015) 1241 [arXiv:1308.4896] [INSPIRE].

[3] D. Honda and T. Okuda, Exact results for boundaries and domain walls in $2 d$ supersymmetric theories, JHEP 09 (2015) 140 [arXiv:1308.2217] [INSPIRE].

[4] K. Hori and M. Romo, Exact results in two-dimensional $(2,2)$ supersymmetric gauge theories with boundary, arXiv:1308.2438 [INSPIRE].

[5] C. Closset, S. Cremonesi and D.S. Park, The equivariant A-twist and gauged linear $\sigma$-models on the two-sphere, JHEP 06 (2015) 076 [arXiv:1504.06308] [INSPIRE].

[6] K. Hori and D. Tong, Aspects of non-abelian gauge dynamics in two-dimensional $\mathcal{N}=(2,2)$ theories, JHEP 05 (2007) 079 [hep-th/0609032] [INSPIRE].

[7] A. Caldararu et al., Non-birational twisted derived equivalences in abelian GLSMs, Commun. Math. Phys. 294 (2010) 605 [arXiv:0709.3855] [INSPIRE].

[8] K. Hori, Duality in two-dimensional $(2,2)$ supersymmetric non-abelian gauge theories, JHEP 10 (2013) 121 [arXiv:1104.2853] [INSPIRE].

[9] H. Jockers et al., Nonabelian 2D gauge theories for determinantal Calabi-Yau varieties, JHEP 11 (2012) 166 [arXiv:1205.3192] [INSPIRE].

[10] R. Donagi and E. Sharpe, GLSM's for partial flag manifolds, J. Geom. Phys. 58 (2008) 1662 [arXiv:0704.1761] [INSPIRE].

[11] K. Hori and J. Knapp, Linear $\sigma$-models with strongly coupled phases - One parameter models, JHEP 11 (2013) 070 [arXiv: 1308.6265] [INSPIRE].

[12] K. Hori and J. Knapp, A pair of Calabi-Yau manifolds from a two parameter non-Abelian gauged linear $\sigma$-model, arXiv: 1612.06214 [INSPIRE].

[13] A. Gerhardus and H. Jockers, Dual pairs of gauged linear $\sigma$-models and derived equivalences of Calabi-Yau threefolds, J. Geom. Phys. 114 (2017) 223 [arXiv:1505.00099] [InSPIRE].

[14] A. Gerhardus and H. Jockers, Quantum periods of Calabi-Yau fourfolds, Nucl. Phys. B 913 (2016) 425 [arXiv: 1604.05325] [INSPIRE].

[15] A. Kanazawa, Pfaffian Calabi-Yau threefolds and mirror symmetry, Commun. Num. Theor. Phys. 6 (2012) 661 [arXiv:1006.0223].

[16] L. Borisov, A. Căldăraru, A. Perry, Intersections of two Grassmannians in $\mathbb{P}^{9}$, arXiv: 1707.00534. 
[17] J. Ottem and J. Rennemo, A counterexample to the birational Torelli problem for Calabi-Yau threefolds, arXiv:1706.09952.

[18] P. Griffiths and J. Harris, Principles of algebraic geometry, John Wiley \& Sons, New York U.S.A. (1978).

[19] J. Harris, Algebraic geometry: a first course, Springer, Germany (1992).

[20] T. Pantev and E. Sharpe, GLSM's for gerbes (and other toric stacks), Adv. Theor. Math. Phys. 10 (2006) 77 [hep-th/0502053] [INSPIRE].

[21] T. Pantev and E. Sharpe, Notes on gauging noneffective group actions, hep-th/0502027 [INSPIRE].

[22] S. Hellerman, A. Henriques, T. Pantev, E. Sharpe and M. Ando, Cluster decomposition, T-duality and gerby CFT's, Adv. Theor. Math. Phys. 11 (2007) 751 [hep-th/0606034] [INSPIRE].

[23] E. Sharpe, Decomposition in diverse dimensions, Phys. Rev. D 90 (2014) 025030 [arXiv: 1404.3986] [INSPIRE].

[24] E. Witten, The Verlinde algebra and the cohomology of the Grassmannian, hep-th/9312104 [INSPIRE].

[25] J. Weyman, Cohomology of vector bundles and syzygies, Cambridge University Press, Cambridge U.K. (2003).

[26] C. van Enckevort and D. van Straten, Electronic data base of Calabi-Yau equations, http://www.mathematik.uni-mainz.de/CYequations/db/.

[27] J. Distler and E. Sharpe, Heterotic compactifications with principal bundles for general groups and general levels, Adv. Theor. Math. Phys. 14 (2010) 335 [hep-th/0701244] [INSPIRE].

[28] E.A. Rødland, The Pfaffian Calabi-Yau, its mirror, and their link to the Grassmannian G(2,7), Composito Math. 122 (2000) 135 [math/9801092].

[29] P.S. Aspinwall and M.R. Plesser, Decompactifications and massless D-branes in hybrid models, JHEP 07 (2010) 078 [arXiv:0909.0252] [INSPIRE].

[30] L. Manivel, Double spinor Calabi-Yau varieties, arXiv:1709.07736.

[31] L. Pertusi, On the double EPW sextic associated to a Gushel-Mukai fourfold, arXiv: 1709.02144.

[32] C. van Enckevort and D. van Straten, Monodromy calculations of fourth order equations of Calabi-Yau type, AMS/IP Stud. Adv. Math. 38 (2006) 539 [math/0412539].

[33] F. Tonoli, Canonical surfaces in $\mathbb{P}^{5}$ and Calabi-Yau threefolds in $\mathbb{P}^{6}$, Ph.D. Thesis, University of Padova, Padova, Italy (2000).

[34] F. Tonoli, Construction of Calabi-Yau 3-folds in $\mathbb{P}^{6}$, J. Alg. Geom. 13 (2004) 209.

[35] S. Cynk and D. van Straten, Calabi-Yau conifold expansions, Fields Inst. Commun. 67 (2018) 499 [arXiv: 1210.3249].

[36] S. Cynk and D. van Straten, Periods of double octic Calabi-Yau manifolds, arXiv: 1709.09751 [INSPIRE].

[37] S. Cynk and D. van Straten, Picard-Fuchs operators for octic arrangements I (The case of orphans), arXiv: 1709.09752.

[38] S. Galkin, Joins and Hadamard products, lecture given at Categorical and analytic invariants in algebraic geometry 1, September 14-18, Stekov Mathematical Institute, Moscow, Russia (2015). 